\title{
MODULAR FORMS ARISING FROM ZETA FUNCTIONS IN TWO VARIABLES ATTACHED TO PREHOMOGENEOUS VECTOR SPACES RELATED TO QUADRATIC FORMS
}

\author{
TAKAHIKO UENO
}

\begin{abstract}
In this paper, we prove the functional equations for the zeta functions in two variables associated with prehomogeneous vector spaces acted on by maximal parabolic subgroups of orthogonal groups. Moreover, applying the converse theorem of Weil type, we show that elliptic modular forms of integral or half integral weight can be obtained from the zeta functions.
\end{abstract}

\section{$\S 1$. Introduction}

The theory of prehomogeneous vector spaces provides a variety of Dirichlet series satisfying functional equations and it is a natural question to ask whether zeta functions of prehomogeneous vector spaces are related to automorphic forms. However we have only few examples of prehomogeneous zeta functions whose relation to automorphic forms are fully understood. In the present paper, generalizing our previous work [14], we consider zeta functions in two variables associated to certain prehomogeneous vector spaces (on which a maximal parabolic subgroup of orthogonal group acts) and prove that, if one of the complex variables takes an integer value, the zeta functions coincide essentially with the Mellin transforms of holomorphic modular forms of one variable.

Our prehomogeneous vector space is the following.

Put $V=\mathbb{C}^{m+2}$ and let $Q(x)$ be a non-degenerate integral quadratic form on $V$ of the form

$$
Q(x)=x_{0} x_{m+1}+\sum_{1 \leq i, j \leq m} a_{i j} x_{i} x_{j},
$$

Received August 13, 2001.

2000 Mathematics Subject Classification: 11F66. 
where $a_{i j}=a_{j i} \in \frac{1}{2} \mathbb{Z}(i \neq j)$ and $a_{i i} \in \mathbb{Z}$. The matrix of $Q$ is given by

$$
\left(\begin{array}{ccc}
0 & 0 & 1 / 2 \\
0 & A & 0 \\
1 / 2 & 0 & 0
\end{array}\right)
$$

with $A=\left(a_{i j}\right)$. We consider the maximal parabolic subgroup of $S O(Q)$ of the form

$$
\left.P=\left\{\begin{array}{ccc}
a & -2 a^{t} u A h & -a A[u] \\
0 & h & u \\
0 & 0 & a^{-1}
\end{array}\right), \begin{array}{c}
a \in \mathbb{C}, a \neq 0 \\
h \in S O(A) \\
u \in \mathbb{C}^{m}
\end{array}\right\}
$$

Then the triple $\left(P \times G L_{1}(\mathbb{C}), V\right)$ is a prehomogeneous vector space.

For positive integers $l, n$, we put

$$
r(l, n)=\sharp\left\{v \in \mathbb{Z}^{m} /(l \mathbb{Z})^{m} \mid A[v] \equiv n(\bmod l)\right\}
$$

and define the Dirichlet series $Z(n, w)$ for $n \in \mathbb{Z}$ (as follows):

$$
Z(n, w)=\sum_{l=1}^{\infty} r(l, n) l^{-w} .
$$

The Dirichlet series $Z(n, w)$ converges absolutely in the domain $\{w \in \mathbb{C} \mid$ $\operatorname{Re}(w)>m\}$. The zeta functions associated with the prehomogeneous vector space is given by

$$
\zeta_{\epsilon}(w, s)=\sum_{n=1}^{\infty} Z(\epsilon n, w) n^{-s} \quad(\epsilon= \pm) .
$$

We prove that the zeta functions have analytic continuations to meromorphic functions on $\mathbb{C}^{2}$ and satisfy functional equations. Let $p$ (resp. $q$ ) be the number of positive (resp. negative) eigenvalues of $A$ and put $c(\epsilon, k)=2 k+1+\epsilon(p-q) / 2$ for $k \in \mathbb{Z}$. We prove that, if we specialize the variable $w$ to

$$
w=c(\epsilon, k)+m / 2>m,
$$

then the zeta function $\zeta_{\epsilon}(w, s)$ is the Mellin transform of a holomorphic modular form of weight $c(\epsilon, k)+1$.

Let $k$ and $N$ be positive integers and $\chi$ a Dirichlet character modulo $N$. We denote by $\mathfrak{G}_{k}(N, \chi)$ the space of holomorphic modular forms of weight 
$k$ and character $\chi$ with respect to $\Gamma_{0}(N)$. When $k$ is odd and $N$ is divisible by 4 , we denote by $G_{k / 2}(N, \chi)$ the space of holomorphic modular forms of weight $k / 2$ and character $\chi$ with respect to $\Gamma_{0}(N)$. Then our main result is the following.

Theorem. Put $D=\operatorname{det} A$ and

$$
a_{\epsilon}(k ; n)= \begin{cases}\frac{(-1)^{k+1}|D|^{1 / 2} \Gamma(c(\epsilon, k)+1) \zeta(c(\epsilon, k)-m / 2+1)}{(2 \pi)^{c(\epsilon, k)+1}} & (n=0), \\ n^{c(\epsilon, k)} Z(\epsilon n, c(\epsilon, k)+m / 2) & (n \geq 1) .\end{cases}
$$

Then the function

$$
f_{\epsilon}(k ; z)=\sum_{n=0}^{\infty} a_{\epsilon}(k ; n) e(n z) \quad(\operatorname{Im} z>0)
$$

is a holomorphic modular form belonging to

$$
\begin{cases}\mathfrak{G}_{c(\epsilon, k)+1}\left(|D|,\left(\frac{(-1)^{m / 2+1} D}{*}\right)\right), & \text { if } m \text { is even and }|D| \neq 2 \bmod 4 \\ \mathfrak{G}_{c(\epsilon, k)+1}\left(4|D|,\left(\frac{(-1)^{m / 2+1} 4 D}{*}\right)\right), & \text { if } m \text { is even and }|D| \equiv 2 \bmod 4 \\ G_{c(\epsilon, k)+1}\left(2|D|, \operatorname{id}_{2|D|}\right), & \text { if } m \text { is odd }\end{cases}
$$

where $\operatorname{id}_{2|D|}$ is the identity character modulo $2|D|$.

The proof of the theorem is based on the functional equations satisfied by $\zeta_{\epsilon}(w, s)$ and the converse theorems of Weil type (Weil [15] and Miyake [3] for the case of integral weight and Shimura [10] for the case of half integral weight).

The zeta functions have been studied in some special cases (see Remark 3 in Section 4). We can regard the prehomogeneous vector space studied in [14] as a special case of the prehomogeneous vector spaces $(P \times$ $\left.G L_{1}(\mathbb{C}), V\right)$, since the group $S L_{2}(\mathbb{C})$ is isomorphic to $\operatorname{Spin}(1,3)$ and is locally isomorphic to $S O(1,3)$. That is the case of $m=2$ and $A$ (negative) definite.

In the course of our investigation, the work [5] of Peter appeared. He studied essentially the same zeta functions under the assumption that $A$ be positive definite. He proved the functional equation of the zeta functions 
from a different view point and conjectured that the zeta functions are related to modular forms. Our theorem give an affirmative answer to his conjecture without any restriction on the signature of $A$. Our approach to the zeta functions is based on the theory of zeta functions in several variables associated with prehomogeneous vector spaces. The use of the general theory of the zeta functions associated with prehomogeneous vector spaces allows us to remove the assumption on the signature and to treat the zeta functions and the twisted zeta functions in a unified manner. We refer to [8] and [9] for a general treatment of $L$-functions of Dirichlet type associated with prehomogeneous vector space.

Now, we give an outline of this paper. In Section 2, we introduce our prehomogeneous vector space and describe the functional equation of local zeta functions associated with the prehomogeneous vector space. In Section 3, we recall the definition of the zeta functions and the zeta integrals associated with the prehomogeneous vector space and give a relation between the zeta functions and the zeta integrals. We also prove the functional equation of zeta integrals and calculate the poles and residues of zeta integrals explicitly. In Section 4, we describe poles and residues of the zeta functions given in Section 3 and prove our main result, Theorem 4.5.

Acknowledgement. I would like to thank Professor F. Sato for helpful advice.

Notation. For $z \in \mathbb{C}$, we use the symbol $e(z)$ as an abbreviation for $\exp (2 \pi \sqrt{-1} z)$ and define $\sqrt{z}=z^{1 / 2}$ so that $-\pi / 2<\arg \left(z^{1 / 2}\right)<\pi / 2$. Further we put $z^{k / 2}=\left(z^{1 / 2}\right)^{k}$ for every $k \in \mathbb{Z}$. For $u \in \mathbb{C}^{m},{ }^{t} u A u$ is abbreviated as $A[u]$.

For any finite dimensional real vector space $V, \mathcal{S}(V)$ is the space of rapidly decreasing $C^{\infty}$-functions on $V$.

\section{\$2. Prehomogeneous vector spaces acted on by maximal parabo- lic subgroups of orthogonal groups}

Let $Q(x)$ be a non-degenerate integral quadratic form on $V=\mathbb{C}^{m+2}$. We assume that $Q(x)$ is of the form

$$
Q(x)=x_{0} x_{m+1}+\sum_{1 \leq i, j \leq m} a_{i j} x_{i} x_{j},
$$


where $a_{i j}=a_{j i} \in \frac{1}{2} \mathbb{Z}(i \neq j)$ and $a_{i i} \in \mathbb{Z}$. The matrix of $Q$ is given by

$$
\left(\begin{array}{ccc}
0 & 0 & 1 / 2 \\
0 & A & 0 \\
1 / 2 & 0 & 0
\end{array}\right)
$$

with $A=\left(a_{i j}\right)$. We assume that the matrix $A$ has $p$ positive and $q$ negative eigenvalues. Let $P$ be the maximal parabolic subgroup of the special orthogonal group $S O(Q)=\left\{g \in G L_{m+2}(\mathbb{C}) \mid Q(g x)=Q(x), \operatorname{det} g=1\right\}$ defined by

$$
\left.P=\left\{\begin{array}{ccc}
a & -2 a^{t} u A h & -a A[u] \\
0 & h & u \\
0 & 0 & a^{-1}
\end{array}\right) \mid \begin{array}{c}
a \in \mathbb{C}, a \neq 0 \\
h \in S O(A) \\
u \in \mathbb{C}^{m}
\end{array}\right\} .
$$

The group $P \times G L_{1}(\mathbb{C})$ acts on $V$ by

$$
\begin{array}{ll}
\rho(p, t) x=t p x & \left(x \in V,(p, t) \in P \times G L_{1}(\mathbb{C})\right), \\
\rho^{*}(p, t) y=t^{-1}{ }^{t} p^{-1} y & \left(y \in V,(p, t) \in P \times G L_{1}(\mathbb{C})\right) .
\end{array}
$$

Then the triples $\left(P \times G L_{1}(\mathbb{C}), \rho, V\right)$ and $\left(P \times G L_{1}(\mathbb{C}), \rho^{*}, V\right)$ are prehomogeneous vector spaces with singular sets

$$
\begin{aligned}
S & =\left\{x \in V \mid x_{m+1}=0\right\} \cup\{x \in V \mid Q(x)=0\}, \\
S^{*} & =\left\{y \in V \mid y_{0}=0\right\} \cup\left\{y \in V \mid Q^{*}(y)=0\right\},
\end{aligned}
$$

where $Q^{*}(y)$ is the quadratic form defined by

$$
Q^{*}(y)=y_{0} y_{m+1}+4^{-1} \sum_{1 \leq i, j \leq m} a_{i j}^{*} y_{i} y_{j} \quad \text { with } A^{-1}=\left(a_{i j}^{*}\right) .
$$

The polynomials $x_{m+1}$ and $Q(x)$ (resp. $y_{0}$ and $\left.Q^{*}(y)\right)$ are the fundamental relative invariants of $\left(P \times G L_{1}(\mathbb{C}), \rho, V\right)\left(\operatorname{resp} .\left(P \times G L_{1}(\mathbb{C}), \rho^{*}, V\right)\right)$ and they satisfy

$$
\begin{array}{ll}
(\rho(p, t) x)_{m+1}=\chi_{1}(p, t) x_{m+1}, & Q(\rho(p, t) x)=\chi(p, t) Q(x), \\
\left(\rho^{*}(p, t) y\right)_{0}=\chi_{1}^{*}(p, t) y_{0}, & Q^{*}\left(\rho^{*}(p, t) y\right)=\chi^{*}(p, t) Q^{*}(y),
\end{array}
$$

where

$$
\begin{aligned}
& \chi_{1}\left(\left(\begin{array}{ccc}
a & * & * \\
0 & h & * \\
0 & 0 & a^{-1}
\end{array}\right), t\right)=t a^{-1}, \quad \chi\left(\left(\begin{array}{ccc}
a & * & * \\
0 & h & * \\
0 & 0 & a^{-1}
\end{array}\right), t\right)=t^{2}, \\
& \chi_{1}^{*}\left(\left(\begin{array}{ccc}
a & * & * \\
0 & h & * \\
0 & 0 & a^{-1}
\end{array}\right), t\right)=(t a)^{-1}, \quad \chi^{*}\left(\left(\begin{array}{ccc}
a & * & * \\
0 & h & * \\
0 & 0 & a^{-1}
\end{array}\right), t\right)=t^{-2} .
\end{aligned}
$$


Hence, we see that $\chi_{1}^{*}(p, t)=\chi_{1} \chi^{-1}(p, t)$ and $\chi^{*}(p, t)=\chi^{-1}(p, t)$. If we identify the dual vector space $V^{*}$ with $V$ by the bilinear form

$$
\langle x, y\rangle=\sum_{i=0}^{m+1} x_{i} y_{i} \quad(x, y \in V)
$$

for $x={ }^{t}\left(x_{0}, x_{1}, \ldots, x_{m+1}\right)$ and $y={ }^{t}\left(y_{0}, y_{1}, \ldots, y_{m+1}\right)$, then the representation $\rho^{*}$ coincides with the contragradient representation of $\rho$.

Put $V_{\mathbb{R}}=\mathbb{R}^{m+2}$ and let

$$
\left.P^{+}=\left\{\begin{array}{ccc}
a & -2 a^{t} u A h & -a A[u] \\
0 & h & u \\
0 & 0 & a^{-1}
\end{array}\right), \begin{array}{c}
a \in \mathbb{R}, a>0 \\
h \in S O(A) \\
u \in \mathbb{R}^{m}
\end{array}\right\} .
$$

Then the $P^{+} \times G L_{1}^{+}(\mathbb{R})$-orbit decomposition of the sets $V_{\mathbb{R}}-S_{\mathbb{R}}$ and $V_{\mathbb{R}}-S_{\mathbb{R}}^{*}$ are given as

$$
V_{\mathbb{R}}-S_{\mathbb{R}}=\bigcup_{\epsilon, \epsilon_{1}} V_{\epsilon \epsilon_{1}}, \quad V_{\mathbb{R}}-S_{\mathbb{R}}^{*}=\bigcup_{\eta, \eta_{1}} V_{\eta \eta_{1}}^{*}
$$

for $\epsilon= \pm, \epsilon_{1}= \pm, \eta= \pm$ and $\eta_{1}= \pm$, where

$$
\begin{aligned}
V_{\epsilon \epsilon_{1}} & =\left\{x \in V_{\mathbb{R}} \mid \operatorname{sgn} Q(x)=\epsilon, \operatorname{sgn} x_{m+1}=\epsilon_{1}\right\}, \\
V_{\eta \eta_{1}}^{*} & =\left\{y \in V_{\mathbb{R}} \mid \operatorname{sgn} Q^{*}(y)=\eta, \operatorname{sgn} y_{0}=\eta_{1}\right\} .
\end{aligned}
$$

For later use, we put $V_{\epsilon}=\bigcup_{\epsilon_{1}} V_{\epsilon \epsilon_{1}}$ and $V_{\eta}^{*}=\bigcup_{\eta_{1}} V_{\eta \eta_{1}}^{*}$. For $f, f^{*} \in \mathcal{S}\left(V_{\mathbb{R}}\right)$, we set

$$
\begin{aligned}
\Phi_{\epsilon \epsilon_{1}}(f ; w, s) & =\int_{V_{\epsilon \epsilon_{1}}}\left|x_{m+1}\right|^{w}|Q(x)|^{s} f(x) d x, \\
\Phi_{\eta \eta_{1}}^{*}\left(f^{*} ; w, s\right) & =\int_{V_{\eta \eta_{1}}^{*}}\left|y_{0}\right|^{w}\left|Q^{*}(y)\right|^{s} f^{*}(y) d y, \\
\Phi_{\epsilon}(f ; w, s) & =\sum_{\epsilon_{1}} \Phi_{\epsilon \epsilon_{1}}(f ; w, s), \\
\Phi_{\eta}^{*}\left(f^{*} ; w, s\right) & =\sum_{\eta_{1}} \Phi_{\eta \eta_{1}}^{*}\left(f^{*} ; w, s\right) .
\end{aligned}
$$

The integrals $\Phi_{\epsilon \epsilon_{1}}(f ; w, s)$ and $\Phi_{\eta \eta_{1}}^{*}\left(f^{*} ; w, s\right)$ converge absolutely for $\operatorname{Re}(w)>0$ and $\operatorname{Re}(s)>0$, so the integrals $\Phi_{\epsilon}$ and $\Phi_{\eta}^{*}$ converge absolutely in the same domain. The integral $\Phi_{\epsilon}(f ; w, s)$ (resp. $\left.\Phi_{\eta}^{*}\left(f^{*} ; w, s\right)\right)$ is called 
the local zeta function associated with the prehomogeneous vector space $\left(P \times G L_{1}(\mathbb{C}), \rho, V\right)\left(\operatorname{resp} .\left(P \times G L_{1}(\mathbb{C}), \rho^{*}, V\right)\right)$.

For $f \in \mathcal{S}\left(V_{\mathbb{R}}\right)$, the Fourier transform $\hat{f}$ of $f$ is given by

$$
\hat{f}(x)=\int_{V_{\mathbb{R}}} f(y) e(x, y) d y,
$$

where we denote $e(\langle x, y\rangle)$ by $e(x, y)$. The local zeta functions $\Phi_{\epsilon}$ and $\Phi_{\eta}^{*}$ satisfy the following functional equations, which was proved in [4] with the method of microlocal calculus and later in [8] with an elementary method.

THEOREM 2.1. The integrals $\Phi_{\epsilon}(f ; w, s)$ and $\Phi_{\eta}^{*}(f ; w, s)$ have analytic continuations to meromorphic functions of $(w, s)$ in $\mathbb{C}^{2}$ and satisfy the following functional equation:

$$
\left(\begin{array}{c}
\Phi_{+} \\
\Phi_{-}
\end{array}\right)(\hat{f} ; w-m, s-1)=\gamma(w, s)\left(\begin{array}{c}
\Phi_{+}^{*} \\
\Phi_{-}^{*}
\end{array}\right)\left(f ; w-m, \frac{m}{2}-w-s\right)
$$

where the $\Gamma$-matrix $\gamma(w, s)$ is given by

$$
\begin{gathered}
\gamma(w, s)=2|D|^{-1 / 2}(2 \pi)^{m / 2-w-2 s} \Gamma(s) \Gamma\left(w+s-\frac{m}{2}\right) \\
\times\left(\begin{array}{cc}
\cos \frac{\pi(w+2 s-p)}{2} & \cos \frac{\pi(w-q)}{2} \\
\cos \frac{\pi(w-p)}{2} & \cos \frac{\pi(w+2 s-q)}{2}
\end{array}\right) .
\end{gathered}
$$

For $f, f^{*} \in \mathcal{S}\left(V_{\mathbb{R}}\right)$, we set

$$
\begin{aligned}
\Sigma(f ; s) & =\int_{\mathbb{R}^{m}} \int_{\mathbb{R}}|a|^{s-m / 2-2} f\left(\begin{array}{c}
-a^{-1} A[u] \\
u \\
a
\end{array}\right) d a d u, \\
\Sigma^{*}\left(f^{*} ; s\right) & =\int_{\mathbb{R}^{m}} \int_{\mathbb{R}}|a|^{s-m / 2-2} f^{*}\left(\begin{array}{c}
a \\
u \\
-(4 a)^{-1} A^{-1}[u]
\end{array}\right) d a d u .
\end{aligned}
$$

The integrals $\Sigma(f ; s)$ and $\Sigma^{*}\left(f^{*} ; s\right)$ converge absolutely for $s \in \mathbb{C}$ with $\operatorname{Re}(s)>(m+2) / 2$.

Proposition 2.2. (1) If $f \in C_{0}^{\infty}\left(V_{\mathbb{R}}-S_{\mathbb{R}}\right)$, we have

$$
\begin{aligned}
\Sigma^{*}(\hat{f} ; s)= & 2|D|^{1 / 2}(2 \pi)^{1-s} \Gamma(s-1) \\
& \times \sum_{\epsilon} \cos \frac{\pi(p-q+\epsilon(2-2 s))}{4} \Phi_{\epsilon}\left(f ; s-\frac{m}{2}-1,1-s\right) .
\end{aligned}
$$


(2) If $f^{*} \in C_{0}^{\infty}\left(V_{\mathbb{R}}-S_{\mathbb{R}}^{*}\right)$, we have

$$
\begin{aligned}
\Sigma\left(\left(f^{*}\right)^{\wedge} ; s\right)= & 2|D|^{-1 / 2}(2 \pi)^{1-s} \Gamma(s-1) \\
& \times \sum_{\eta} \cos \frac{\pi(q-p+\eta(2-2 s))}{4} \Phi_{\eta}^{*}\left(f^{*} ; s-\frac{m}{2}-1,1-s\right) .
\end{aligned}
$$

Let us recall the following formulas for the Fourier transforms of $|x|^{s}$ and $e(A[u])$ for a symmetric matrix with $\operatorname{sgn} A=(p, q)$ which are necessary for the proof of Proposition 2.2:

$$
\begin{aligned}
& \left(\begin{array}{l}
\int_{0}^{\infty}|x|^{s-1} \hat{f}(x) d x \\
\int_{-\infty}^{0}|x|^{s-1} \hat{f}(x) d x
\end{array}\right) \\
& =(2 \pi)^{-s} \Gamma(s)\left(\begin{array}{cc}
e\left(\frac{s}{4}\right) & e\left(-\frac{s}{4}\right) \\
e\left(-\frac{s}{4}\right) & e\left(\frac{s}{4}\right)
\end{array}\right)\left(\begin{array}{c}
\int_{0}^{\infty}|x|^{-s} f(x) d x \\
\int_{-\infty}^{0}|x|^{-s} f(x) d x
\end{array}\right), \\
& \int_{\mathbb{R}^{m}} \hat{f}(u) e(A[u]) d u \\
& \quad=|\operatorname{det} 2 A|^{-1 / 2} e\left(\frac{p-q}{8}\right) \int_{\mathbb{R}^{m}} f(u) e\left(-\frac{1}{4} A^{-1}[u]\right) d u
\end{aligned}
$$

Proof of Proposition 2.2. We prove only (1), since the second assertion is proved in the same way. By the principle of analytic continuation, one may assume $\operatorname{Re}(s)>(m+2) / 2$. Then, since $f \in C_{0}^{\infty}\left(V_{\mathbb{R}}-S_{\mathbb{R}}\right)$, the integrals that will appear in the calculation below are absolutely convergent. We have

$$
\begin{aligned}
\Sigma^{*}(\hat{f} ; s)= & \int_{\mathbb{R}^{m}} \int_{\mathbb{R}}|a|^{s-m / 2-2} \hat{f}\left(\begin{array}{c}
a \\
u \\
-(4 a)^{-1} A^{-1}[u]
\end{array}\right) d a d u \\
= & \int_{\mathbb{R}}|a|^{s-m / 2-2} d a \int_{\mathbb{R}^{m}} d u \\
& \times \int_{V_{\mathbb{R}}} f(x) e\left(a x_{0}+{ }^{t} u v-4^{-1} a^{-1} x_{m+1} A^{-1}[u]\right) d x_{0} d v d x_{m+1} \\
= & \int_{\mathbb{R}}|a|^{s-m / 2-2} d a \int_{\mathbb{R}^{2}} d x_{0} d x_{m+1} \int_{\mathbb{R}^{m}} e\left(-\frac{x_{m+1}}{4 a} A^{-1}[u]\right) d u \\
& \times \int_{\mathbb{R}^{m}}\left\{f(x) e\left(a x_{0}\right)\right\} e\left({ }^{t} u v\right) d v
\end{aligned}
$$


for $x={ }^{t}\left(x_{0},{ }^{t} v, x_{m+1}\right)$. Using the formula (2.4), we see that

$$
\begin{aligned}
& \Sigma^{*}(\hat{f} ; s)=|D|^{1 / 2} \int_{\mathbb{R}}|a|^{s-2} d a \int_{V_{\mathbb{R}}}\left|x_{m+1}\right|^{-m / 2} f(x) e\left(a x_{m+1}^{-1} Q(x)\right) \\
& \quad \times e\left(\frac{(q-p) \operatorname{sgn}\left(a x_{m+1}\right)}{8}\right) d x_{0} d v d x_{m+1} \\
& =|D|^{1 / 2} \sum_{\epsilon} \sum_{\eta} e\left(\frac{\epsilon \eta(q-p)}{8}\right) \int_{\operatorname{sgn}(a)=\epsilon}|a|^{s-2} d a \\
& \quad \times \int_{\operatorname{sgn}\left(x_{m+1}\right)=\eta}\left|x_{m+1}\right|^{-m / 2} d x_{m+1} \int_{\mathbb{R}^{m+1}} f(x) e\left(a x_{m+1}^{-1} Q(x)\right) d x_{0} d v
\end{aligned}
$$

Put $y_{0}=x_{m+1}^{-1} Q(x)$. Then, since $\operatorname{sgn}\left(y_{0}\right)=\eta \operatorname{sgn}(Q(x))$ and $x_{0}=y_{0}-$ $x_{m+1}^{-1} A[v]$, we have

$$
\begin{aligned}
\Sigma^{*}(\hat{f} ; s)=|D|^{1 / 2} \sum_{\epsilon} \sum_{\eta} e\left(\frac{\epsilon \eta(q-p)}{8}\right) \int_{\operatorname{sgn}(a)=\epsilon}|a|^{s-2} d a \\
\quad \times \int_{\operatorname{sgn}\left(x_{m+1}\right)=\eta}\left|x_{m+1}\right|^{-m / 2} d x_{m+1} \int_{\mathbb{R}^{m+1}} f\left(x^{\prime}\right) e\left(a y_{0}\right) d y_{0} d v
\end{aligned}
$$

for $x^{\prime}={ }^{t}\left(y_{0}-x_{m+1}^{-1} A[v],{ }^{t} v, x_{m+1}\right)$. By the formula (2.3), we have

$$
\begin{aligned}
\Sigma^{*}(\hat{f} ; s)=2|D|^{1 / 2}(2 \pi)^{1-s} \Gamma(s-1) \sum_{\eta} \int_{\operatorname{sgn}\left(x_{m+1}\right)=\eta}\left|x_{m+1}\right|^{-m / 2} d x_{m+1} \\
\quad \times \int_{\mathbb{R}^{m+1}} \cos \frac{\eta \pi(q-p-\operatorname{sgn}(Q(x))(2-2 s))}{4}\left|y_{0}\right|^{1-s} f\left(x^{\prime}\right) d y_{0} d v \\
=2|D|^{1 / 2}(2 \pi)^{1-s} \Gamma(s-1) \int_{V_{\mathbb{R}}}\left|x_{m+1}\right|^{s-m / 2-1}|Q(x)|^{1-s} \\
\times f(x) \cos \frac{\pi(q-p-\operatorname{sgn}(Q(x))(2-2 s))}{4} d x_{0} d v d x_{m+1} .
\end{aligned}
$$

Therefore we obtain

$$
\begin{aligned}
\Sigma^{*}(\hat{f} ; s-1)= & 2|D|^{1 / 2}(2 \pi)^{1-s} \Gamma(s-1) \\
& \times\left(\cos \frac{\pi(q-p-2+2 s)}{4} \Phi_{+}(f ; s-m / 2-1,1-s)\right. \\
& \left.\quad+\cos \frac{\pi(q-p+2-2 s)}{4} \Phi_{-}(f ; s-m / 2-1,1-s)\right) .
\end{aligned}
$$




\section{§3. Zeta functions associated with the prehomogeneous vector space}

In this section, we define the zeta functions and the zeta integrals associated with the prehomogeneous vector space $\left(P \times G L_{1}(\mathbb{C}), \rho, V\right)$ and describe a relation between the zeta functions and the zeta integrals. Moreover, we prove the functional equation of zeta integrals. For later use, we also consider the zeta functions twisted by Dirichlet characters with prime conductors.

In the following, we consider the group

$$
G=\left\{\left(\begin{array}{ccc}
a & -2 a^{t} u A & -a A[u] \\
0 & 1_{m} & u \\
0 & 0 & a^{-1}
\end{array}\right) \mid \begin{array}{c}
a \in \mathbb{C}, a \neq 0 \\
u \in \mathbb{C}^{m}
\end{array}\right\} \times G L_{1}(\mathbb{C})
$$

instead of $P \times G L_{1}$. Then the triple $(G, \rho, V)$ remains a prehomogeneous vector space and the relative invariants are the same as in Section 2. Moreover, it turns out that the zeta functions are essentially the same and it is easier to treat the zeta functions if we regard them as zeta functions associated to $(G, \rho, V)$.

We put

$$
\begin{aligned}
G^{+} & =\left\{\left(\begin{array}{ccc}
a & -2 a^{t} u A & -a A[u] \\
0 & 1_{m} & u \\
0 & 0 & a^{-1}
\end{array}\right) \mid \begin{array}{c}
a \in \mathbb{R}, a>0 \\
u \in \mathbb{R}^{m}
\end{array}\right\} \times G L_{1}^{+}(\mathbb{R}), \\
\Gamma & =\left\{\left(\begin{array}{ccc}
1 & -2^{t} u A & -A[u] \\
0 & 1_{m} & u \\
0 & 0 & 1
\end{array}\right) \mid u \in \mathbb{Z}^{m}\right\}, \\
V_{\mathbb{Q}} & =\mathbb{Q}^{m+2}, \quad V_{\mathbb{Z}}=\mathbb{Z}^{m+2} .
\end{aligned}
$$

Let $r$ be an odd prime number and $\psi$ a Dirichlet character modulo $r$. We understand that $\psi(n)=0$ if $(r, n) \neq 1$. We define the following functions on $V_{\mathbb{Q}}$ :

$$
\phi_{1}(x)=\left\{\begin{array}{ll}
1 & \text { if } x \in V_{\mathbb{Z}}, \\
0 & \text { if } x \notin V_{\mathbb{Z}},
\end{array} \quad \phi_{\psi}(x)= \begin{cases}\psi(Q(x)) & \text { if } x \in V_{\mathbb{Z}}, \\
0 & \text { if } x \notin V_{\mathbb{Z}} .\end{cases}\right.
$$

Since the polynomial $Q(x)$ is $\Gamma$-invariant, the functions $\phi_{1}$ and $\phi_{\psi}$ are $\Gamma$ invariant. 
For a function $\phi=\phi_{1}$ (or $\phi_{\psi}$ ) and each $y \in V_{\mathbb{Q}}$, we take a positive integer $M$, which may depend on $y$, such that

$$
x \equiv x^{\prime}\left(\bmod M V_{\mathbb{Z}}\right) \Longrightarrow \phi(x) e(-x, y)=\phi\left(x^{\prime}\right) e\left(-x^{\prime}, y\right) \text {. }
$$

Define $\hat{\phi}$ as follows:

$$
\hat{\phi}(y)=M^{-m-2} \sum_{x \in V_{\mathbb{Q}} / M V_{\mathbb{Z}}} \phi(x) e(-x, y), \quad\left(y \in V_{\mathbb{Q}}\right) .
$$

This definition does not depend on the choice of $M$. We call the function $\hat{\phi}$ the Fourier transform of $\phi$. These functions appear in the functional equation of the zeta functions.

In the following, the "quadratic residue symbol" $\left(\frac{a}{b}\right)$ has the same meaning as in [10].

For an odd positive integer $r$, we put $\epsilon_{r}=1$ or $\sqrt{-1}$ according as $r \equiv 1$ or $3(\bmod 4)$. For a Dirichlet character $\psi$ modulo $N$, we denote by $\psi_{(N)}$ and $\psi^{(N)}$ respectively, $\left(\frac{*}{N}\right) \psi$ and $\left(\frac{N}{*}\right) \psi$. For a primitive Dirichlet character $\psi$ modulo $N$, the Gauss sum $\tau(\psi)$ is given by

$$
\tau(\psi):=\sum_{j=1}^{N} \psi(j) e(j / N)
$$

The following proposition gives the Fourier transform of $\phi$.

Proposition 3.1. (1) We have

$$
\hat{\phi}_{1}(y)= \begin{cases}1 & \text { if } y \in V_{\mathbb{Z}} \\ 0 & \text { if } y \notin V_{\mathbb{Z}}\end{cases}
$$

(2) Let $r$ be a prime number not dividing $2|D|, \psi$ a primitive Dirichlet character modulo $r$ and put $C_{r, m}=r^{-m / 2-1} \epsilon_{r}^{m+2}$.

(i) If $m$ is even, then we have

$$
\hat{\phi}_{\psi}(y)= \begin{cases}C_{r, m}\left(\frac{D}{r}\right) \psi(-D) \tau(\psi) \tau(\bar{\psi})^{-1} \bar{\psi}\left(D Q^{*}(r y)\right) & \text { if } y \in r^{-1} V_{\mathbb{Z}}, \\ 0 & \text { if } y \notin r^{-1} V_{\mathbb{Z}} .\end{cases}
$$


(ii) If $m$ is odd and $\psi \neq\left(\frac{*}{r}\right)$, then we have

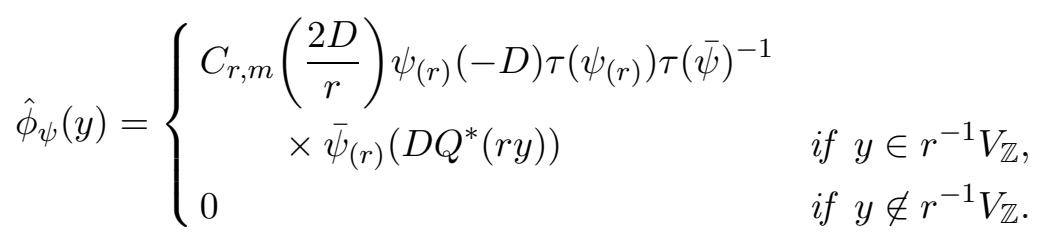

(iii) If $m$ is odd and $\psi=\left(\frac{*}{r}\right)$, then we have

$$
\hat{\phi}_{\psi}(y)= \begin{cases}C_{r, m} \alpha_{\left(\frac{*}{r}\right)} & \text { if } y \in r^{-1} V_{\mathbb{Z}}, \\ 0 & \text { if } y \notin r^{-1} V_{\mathbb{Z}},\end{cases}
$$

where

$$
\alpha_{\left(\frac{*}{r}\right)}= \begin{cases}(r-1) \times\left(\frac{2}{r}\right)^{m+2}\left(\frac{D}{r}\right) \epsilon_{r}^{-1} r^{-1 / 2} & \text { if } r \mid Q^{*}(r y), \\ -\left(\frac{2}{r}\right)^{m+2}\left(\frac{D}{r}\right) \epsilon_{r}^{-1} r^{-1 / 2} & \text { if } r \nmid Q^{*}(r y) .\end{cases}
$$

Remark 1. (1) If $m$ is odd, then $|D|$ is even and $2|D| Q^{*}$ has integral coefficients. If $m$ is even, then $|D| Q^{*}$ has integral coefficients (Remark 4 and Lemma 4 in [13]).

(2) The character $\left(\frac{D}{*}\right)$ is a character of modulo $4|D|$ if $m$ is even and $|D| \equiv 2 \bmod 4$.

For the proof of the above proposition, we need the following lemma. The first assertion of the lemma is due to Stark [13, Theorem 1] and the second assertion can be proved easily by using the identity (36) in [13].

LEMMA 3.2. Let $q(x)$ be an n-variable non-degenerate quadratic form with integral coefficients, $F$ the symmetric matrix with $F[x]=2 q(x), d=$ $\operatorname{det} F$ and $\bar{q}(y)=2^{-1} d F^{-1}[y]$. Let $r$ be an odd prime number not dividing $d$ and $\psi$ a primitive Dirichlet character modulo $r$.

(1) If $n$ is even or $\psi \neq\left(\frac{*}{r}\right)$, then we have

$$
\sum_{x \in(\mathbb{Z} / r \mathbb{Z})^{m}} \psi(q(x)) e\left(r^{-1}\langle x, y\rangle\right)=\alpha_{\psi} \tau(\bar{\psi})^{-1} r^{n / 2} \bar{\psi}^{\prime}(\bar{q}(y)),
$$


where

$$
\alpha_{\psi}=\left(\epsilon_{r}\left(\frac{2}{r}\right)\right)^{n}\left(\frac{d}{r}\right) \psi^{\prime}(-d) \tau\left(\psi^{\prime}\right), \quad \psi^{\prime}(j)=\psi(j)\left(\frac{j}{r}\right)^{n}
$$

(2) If $n$ is odd, then we have

$$
\begin{aligned}
& \sum_{x \in(\mathbb{Z} / r \mathbb{Z})^{m}}\left(\frac{q(x)}{r}\right) e\left(r^{-1}\langle x, y\rangle\right) \\
& = \begin{cases}\epsilon_{r}^{n-1}\left(\frac{2 d}{r}\right)(r-1) r^{(n-1) / 2} & \text { if } r \mid \bar{q}(y), \\
-\epsilon_{r}^{n-1}\left(\frac{2 d}{r}\right) r^{(n-1) / 2} & \text { if } r \nmid \bar{q}(y) .\end{cases}
\end{aligned}
$$

Proof of Proposition 3.1. We only prove the second assertion, since the first assertion can be proved in the same way. For $y={ }^{t}\left(y_{0}, y_{1}, \ldots, y_{m+1}\right) \in$ $V_{\mathbb{Q}}$, we can take a positive integer $q$ such that $q y_{i} \in \mathbb{Z}$ for $0 \leq i \leq m+1$. By the definition of the function $\hat{\phi}_{\psi}$, we have

$$
\begin{aligned}
\hat{\phi}_{\psi}(y) & =(q r)^{-m-2} \sum_{x \in V_{\mathbb{Q}} / q r V_{\mathbb{Z}}} \phi_{\psi}(x) e(-x, y) \\
& =(q r)^{-m-2} \sum_{a \bmod r V_{\mathbb{Z}}} \psi(Q(a)) e(a, y) \sum_{b \bmod q V_{\mathbb{Z}}} e(r b, y) .
\end{aligned}
$$

Since

$$
\sum_{b \bmod q V_{\mathbb{Z}}} e(r b, y)= \begin{cases}q^{m+2} & \text { if } y \in r^{-1} V_{\mathbb{Z}}, \\ 0 & \text { if } y \notin r^{-1} V_{\mathbb{Z}}\end{cases}
$$

we see that the function $\hat{\phi}_{\psi}(y)$ becomes

$$
\hat{\phi}_{\psi}(y)= \begin{cases}r^{-m-2} \sum_{a \bmod r V_{\mathbb{Z}}} \psi(Q(a)) e(a, y) & \text { if } y \in r^{-1} V_{\mathbb{Z}}, \\ 0 & \text { if } y \notin r^{-1} V_{\mathbb{Z}}\end{cases}
$$

Hence our assertion follows from Proposition 3.2.

Now we introduce the zeta integrals and the (twisted) zeta functions associated with the prehomogeneous vector space $(G, \rho, V)$. 
For $f, f^{*} \in \mathcal{S}\left(V_{\mathbb{R}}\right)$ and $\phi=\phi_{1}$ or $\phi_{\psi}$, the zeta integrals $Z(f, \phi ; w, s)$ and $Z^{*}\left(f^{*}, \hat{\phi} ; w, s\right)(w, s \in \mathbb{C})$ are given by

$$
\begin{aligned}
Z(f, \phi ; w, s) & :=\int_{G^{+} / \Gamma} \chi_{1}(p, t)^{w} \chi(p, t)^{s} \sum_{x \in V_{\mathbb{Q}}-S_{\mathbb{Q}}} \phi(x) f(\rho(p, t) x) d_{r} g, \\
Z^{*}\left(f^{*}, \hat{\phi} ; w, s\right) & :=\int_{G^{+} / \Gamma} \chi_{1}^{*}(p, t)^{w} \chi^{*}(p, t)^{s} \sum_{x \in V_{\mathbb{Q}}-S_{\mathbb{Q}}^{*}} \hat{\phi}(x) f^{*}\left(\rho^{*}(p, t) x\right) d_{r} g,
\end{aligned}
$$

where $d_{r} g$ is the right-invariant measure on $G^{+}$normalized by

$$
d_{r} g=2 t^{-1} a^{m-1} d t d a d u \quad \text { for } g=\left(\left(\begin{array}{ccc}
a & -2 a^{t} u A & -a A[u] \\
0 & 1_{m} & u \\
0 & 0 & a^{-1}
\end{array}\right), t\right) .
$$

The zeta functions $\zeta_{\epsilon \epsilon_{1}}(\phi ; w, s)$ and $\zeta_{\eta \eta_{1}}^{*}(\hat{\phi} ; w, s)$ are defined for $\epsilon, \epsilon_{1}, \eta, \eta_{1}=$ \pm by

$$
\begin{aligned}
\zeta_{\epsilon \epsilon_{1}}(\phi ; w, s) & :=\sum_{x \in \Gamma \backslash V_{\epsilon \epsilon_{1}} \cap V_{\mathbb{Q}}} \phi(x)\left|x_{m+1}\right|^{-w}|Q(x)|^{-s}, \\
\zeta_{\eta \eta_{1}}^{*}(\hat{\phi} ; w, s) & :=\sum_{y \in \Gamma \backslash V_{\eta \eta_{1}}^{*} \cap V_{\mathbb{Q}}} \hat{\phi}(y)\left|y_{0}\right|^{-w}\left|Q^{*}(y)\right|^{-s},
\end{aligned}
$$

where $V_{\epsilon \epsilon_{1}}$ and $V_{\eta \eta_{1}}^{*}$ are given by (2.1) and (2.2), respectively. It is obvious that $\zeta_{\epsilon+}=\zeta_{\epsilon-}$ and $\zeta_{\eta+}^{*}=\zeta_{\eta-}^{*}$, so we put

$$
\begin{aligned}
\zeta_{\epsilon}(\phi ; w, s) & :=\zeta_{\epsilon+}(\phi ; w, s) \\
\zeta_{\eta}^{*}(\hat{\phi} ; w, s) & :=\zeta_{\eta+}^{*}(\hat{\phi} ; w, s) .
\end{aligned}
$$

It is easy to see that our prehomogeneous vector space satisfies the condition of Theorem 1 in [7] and the zeta functions converge absolutely in $\operatorname{Re}(w)>$ $m, \operatorname{Re}(s)>1$.

Next we define the Dirichlet series $Z(n, w)$ and $Z^{*}(n, w)$ for $n \in \mathbb{Z}$, which are related to the zeta functions above:

$$
Z(n, w)=\sum_{l=1}^{\infty} \frac{r(l, n)}{l^{w}}, \quad Z^{*}(n, w)=\sum_{l=1}^{\infty} \frac{r^{*}(l, n)}{l^{w}},
$$

where

$$
\begin{aligned}
r(l, n) & =\sharp\left\{v \in \mathbb{Z}^{m} /(l \mathbb{Z})^{m} \mid A[v] \equiv n(\bmod l)\right\}, \\
r^{*}(l, n) & =\sharp\left\{v^{*} \in \mathbb{Z}^{m} / 2 l A \mathbb{Z}^{m}\left|2^{-1} \cdot\right| D \mid A^{-1}\left[v^{*}\right] \equiv n(\bmod 2|D| l)\right\}
\end{aligned}
$$


if $m$ is odd,

$$
r^{*}(l, n)=\sharp\left\{v^{*} \in \mathbb{Z}^{m} / 2 l A \mathbb{Z}^{m}\left|4^{-1} \cdot\right| D \mid A^{-1}\left[v^{*}\right] \equiv n(\bmod |D| l)\right\}
$$

if $m$ is even.

Then we see that the Dirichlet series $Z(n, w)$ and $Z^{*}(n, w)$ converge absolutely for $\operatorname{Re}(w) \gg 0$ from simple estimates of $r(l, n)$ and $r^{*}(l, n)$. Moreover, from Lemmas 3.3, 3.4 and the convergence of the zeta functions, we can show that $Z(n, w)$ and $Z^{*}(n, w)$ converge absolutely in the domain $\{w \in \mathbb{C} \mid \operatorname{Re}(w)>m\}$. The Dirichlet series $Z(n, w)$ coincides with the series $L(w, n ; 1, A)$ studied in [5], where the following lemma is proved.

Lemma 3.3. (1) ([5, Lemma 3.8]) For $n \in \mathbb{Z} \backslash\{0\}$ define

$$
F(n, w):=\frac{Z(n, w) L\left(w-\frac{m}{2}+1,\left(\frac{(-1)^{m / 2+1} 4 D}{*}\right)\right)}{\zeta(w-m+1)}
$$

if $m$ is even and

$$
F(n, w):=\frac{(w-(m+1) / 2) Z(n, w) \zeta(2 w-m+1)}{\zeta(w-m+1)} \prod_{p \mid 2 D}\left(1-p^{m-1-2 w}\right)
$$

if $m$ is odd. Then $F(n, w)$ can be extended to an entire function. For $\sigma_{1} \in \mathbb{R}$ there exist constants $c_{1}, c_{2}>0$ such that

$$
|F(n, w)| \leq c_{1}|n|^{c_{2}}(|\operatorname{Im}(w)|+1)^{c_{2}}(|w|+1) \quad \text { for } \operatorname{Re}(w) \geq \sigma_{1}
$$

(2) ([5, Lemma 3.9]) Define

$$
F(0, w):=\frac{Z(0, w) L\left(w-\frac{m}{2}+1,\left(\frac{(-1)^{m / 2+1} 4 D}{*}\right)\right)}{\zeta(w-m+1) L\left(w-\frac{m}{2},\left(\frac{(-1)^{m / 2+1} 4 D}{*}\right)\right)} \prod_{p \mid 2 D}\left(1-p^{m-2 w}\right)
$$

if $m$ is even and

$$
F(0, w):=\frac{Z(0, w) \zeta(2 w-m+1)}{\zeta(2 w-m) \zeta(w-m+1)} \prod_{p \mid 2 D}\left(1-p^{m-1-2 w}\right)
$$

if $m$ is odd. Then $F(0, w)$ can be extended to an entire function. For $-\infty<\sigma_{1}<\sigma_{2}<\infty$ there exists a constant $c>0$ such that $|F(0, w)| \leq c$ for $\sigma_{1} \leq \operatorname{Re}(w) \leq \sigma_{2}$. 
The functions $Z(n, w), Z^{*}(n, w), \zeta_{\epsilon}(\phi ; w, s)$ and $\zeta_{\eta}^{*}(\hat{\phi} ; w, s)$ satisfy the following relation.

LEMMA 3.4. (1) If $m$ is even, then we have

$$
\begin{aligned}
& \zeta_{\epsilon}\left(\phi_{1} ; w, s\right)=\sum_{n=1}^{\infty} Z(\epsilon n, w) n^{-s} \\
& \zeta_{\eta}^{*}\left(\hat{\phi}_{1} ; w, s\right)= \begin{cases}|D|^{s} \sum_{n=1}^{\infty} Z^{*}(\eta n, w) n^{-s} & \text { if }|D| \neq 2(\bmod 4), \\
(4|D|)^{s} \sum_{n=1}^{\infty} Z^{*}(\eta n, w)(4 n)^{-s} & \text { if }|D| \equiv 2(\bmod 4),\end{cases} \\
& \zeta_{\epsilon}\left(\phi_{\psi} ; w, s\right)=\psi(\epsilon) \sum_{n=1}^{\infty} \psi(n) Z(\epsilon n, w) n^{-s}, \\
& \zeta_{\eta}^{*}\left(\hat{\phi}_{\psi} ; w, s\right)=c(\psi, D, \eta) r^{w-m / 2-1} \\
& \times \begin{cases}\left(|D| r^{2}\right)^{s} \sum_{n=1}^{\infty} \bar{\psi}(n) Z^{*}(\eta n, w) n^{-s} & \text { if }|D| \neq \equiv 2(\bmod 4), \\
\left(4|D| r^{2}\right)^{s} \sum_{n=1}^{\infty} \bar{\psi}(4 n) Z^{*}(\eta n, w)(4 n)^{-s} & \text { if }|D| \equiv 2(\bmod 4),\end{cases}
\end{aligned}
$$

where

$$
\begin{aligned}
c(\psi, D, \eta)=\frac{\epsilon_{r}^{m+2}\left(\frac{D}{r}\right) \psi(-D) \tau(\psi)}{\tau(\bar{\psi})} & \text { if }|D| \neq 2(\bmod 4), \\
& \times \begin{cases}\bar{\psi}(\eta \operatorname{sgn}(D)) & \text { if }|D| \equiv 2(\bmod 4) . \\
\bar{\psi}\left(4^{-1} \eta \operatorname{sgn}(D)\right) & \text { if } \mid D\end{cases}
\end{aligned}
$$

(2) If $m$ is odd, then we have

$$
\begin{aligned}
\zeta_{\epsilon}\left(\phi_{1} ; w, s\right) & =\sum_{n=1}^{\infty} Z(\epsilon n, w) n^{-s} \\
\zeta_{\eta}^{*}\left(\hat{\phi}_{1} ; w, s\right) & =(2|D|)^{s} \sum_{n=1}^{\infty} Z^{*}(\eta n, w) n^{-s},
\end{aligned}
$$




$$
\begin{aligned}
& \zeta_{\epsilon}\left(\phi_{\psi} ; w, s\right)=\psi(\epsilon) \sum_{n=1}^{\infty} \psi(n) Z(\epsilon n, w) n^{-s}, \\
& \zeta_{\eta}^{*}\left(\hat{\phi}_{\psi} ; w, s\right)=c(\psi, D, \eta) r^{w-m / 2-1}\left(2|D| r^{2}\right)^{s} \\
& \quad \times \begin{cases}\sum_{n=1}^{\infty} \bar{\psi}_{(r)}(n) Z^{*}(\eta n, w) n^{-s} & \text { if } \psi \neq\left(\frac{*}{r}\right), \\
\left(\sum_{n=1}^{\infty} r Z^{*}(\eta r n, w)(r n)^{-s}-\sum_{n=1}^{\infty} Z^{*}(\eta n, w) n^{-s}\right) & \text { if } \psi=\left(\frac{*}{r}\right),\end{cases}
\end{aligned}
$$

where

$$
\begin{aligned}
& c(\psi, D, \eta) \\
& = \begin{cases}\bar{\psi}_{(r)}(\eta \operatorname{sgn}(D)) \epsilon_{r}^{m+2}\left(\frac{2 D}{r}\right) \psi_{(r)}(-2 D) \tau\left(\psi_{(r)}\right) \tau(\bar{\psi})^{-1} & \text { if } \psi \neq\left(\frac{*}{r}\right), \\
\epsilon_{r}^{m+1}\left(\frac{2 D}{r}\right) r^{-1 / 2} & \text { if } \psi=\left(\frac{*}{r}\right) .\end{cases}
\end{aligned}
$$

Remark 2. (1) Lemma 3.4 shows that the zeta functions for $\phi_{1}$ and $\hat{\phi}_{1}$ coincide up to an elementary factor with the Dirichlet series $\tilde{\tau}$ studied in [5], where $A$ is assumed to be positive definite.

(2) Since $\epsilon_{r}^{2}=\left(\frac{-1}{r}\right)$, we obtain

$$
\begin{aligned}
& c(\psi, D, \eta) \\
& = \begin{cases}\bar{\psi}(\eta)\left(\frac{(-1)^{m / 2+1} D}{r}\right) \psi(-|D|) \tau(\psi) / \tau(\bar{\psi}) & \text { if }|D| \not \equiv 2(\bmod 4), \\
\bar{\psi}(\eta)\left(\frac{(-1)^{m / 2+1} D}{r}\right) \psi(-4|D|) \tau(\psi) / \tau(\bar{\psi}) & \text { if }|D| \equiv 2(\bmod 4),\end{cases}
\end{aligned}
$$

in the above lemma (1). In the above lemma $(2), \operatorname{since} \operatorname{sgn}(D)=(-1)^{q+1}$, we obtain

$$
\begin{aligned}
& c(\psi, D, \eta) \\
& = \begin{cases}\bar{\psi}(\eta)\left(\frac{\eta}{r}\right) \epsilon_{r}^{p-q+2}\left(\frac{2 D}{r}\right) \psi_{r}(-2|D|)\left(\frac{2 D}{r}\right) \tau\left(\psi_{(r)}\right) / \tau(\bar{\psi}) & \text { if } \psi \neq\left(\frac{*}{r}\right), \\
\epsilon_{r}^{p-q-1}\left(\frac{2 D}{r}\right) r^{-1 / 2} & \text { if } \psi=\left(\frac{*}{r}\right) .\end{cases}
\end{aligned}
$$


Proposition 3.5. The integrals $Z(f, \phi ; w, s), Z^{*}\left(f^{*}, \hat{\phi} ; w, s\right)$ and the Dirichlet series $\zeta_{\epsilon}(\phi ; w, s), \zeta_{\eta}^{*}(\hat{\phi} ; w, s)$ are absolutely convergent for $\operatorname{Re}(w)>$ $m, \operatorname{Re}(s)>1$. Moreover, the following equalities hold:

$$
\begin{aligned}
Z(f, \phi ; w, s) & =\sum_{\epsilon} \zeta_{\epsilon}(\phi ; w, s) \Phi_{\epsilon}(f ; w-m, s-1), \\
Z^{*}\left(f^{*}, \hat{\phi} ; w, s\right) & =\sum_{\eta} \zeta_{\eta}^{*}(\hat{\phi} ; w, s) \Phi_{\eta}^{*}\left(f^{*} ; w-m, s-1\right) .
\end{aligned}
$$

Proof. For any $x_{0} \in V_{\epsilon \epsilon 1}, G_{x_{0}}^{+}$is trivial and

$$
\int_{G^{+}} F\left(\rho(g) x_{0}\right) d_{r} g=\int_{V_{\epsilon \epsilon_{1}}} F(x) \omega(x) \text { for } F \in L^{1}\left(V_{\epsilon \epsilon_{1}}, \omega\right),
$$

where $\omega(x)=\left|x_{m+1}\right|^{-m}|Q(x)|^{-1} d x$, which is a $G^{+}$-relative invariant measure on $V_{\mathbb{R}}$ and the space $L^{1}\left(V_{\epsilon \epsilon_{1}}, \omega\right)$ is the vector space of absolutely $\omega$ integrable functions. Using the formula (3.1), we can show that

$$
Z(f, \phi ; w, s)=\sum_{\epsilon} \Phi_{\epsilon}(f ; w-m, s-1) \zeta_{\epsilon}(\phi ; w, s) .
$$

Since the series $\zeta_{\epsilon}(\phi ; w, s)$ converges for $\operatorname{Re}(w)>m, \operatorname{Re}(s)>1$, our assertion is proved.

We define the integrals $Z_{+}(f, \phi ; w, s)$ and $Z_{+}^{*}\left(f^{*}, \phi ; w, s\right)$ by putting

$$
\begin{aligned}
Z_{+}(f, \phi ; w, s)= & \int_{G_{\mathbb{R}} / \Gamma, \chi(p, t) \geq 1} \chi_{1}(p, t)^{w} \chi(p, t)^{s} \\
\times \sum_{x \in V_{\mathbb{Q}}-S_{\mathbb{Q}}} \phi(x) f(\rho(p, t) x) d_{r} g, & \int_{G_{\mathbb{R}} / \Gamma, \chi^{*}(p, t) \geq 1} \chi_{1}^{*}(p, t)^{w} \chi^{*}(p, t)^{s} \\
& \times \sum_{x \in V_{\mathbb{Q}}-S_{\mathbb{Q}}^{*}}^{*} \phi\left(f^{*}, \phi ; w, s\right) f^{*}\left(\rho^{*}(p, t) x\right) d_{r} g .
\end{aligned}
$$

Proposition 3.5 implies that the integrals $Z_{+}(f, \phi ; w, s)$ and $Z_{+}^{*}\left(f^{*}, \phi ; w, s\right)$ are holomorphic functions in the domain $\left\{(w, s) \in \mathbb{C}^{2} \mid \operatorname{Re}(w)>m\right\}$. $\mathcal{S}\left(V_{\mathbb{R}}\right)$.

Proposition 3.6. Assume that $\operatorname{Re}(w)>m, \operatorname{Re}(s)>1$ and $f \in$ 
(1) We have

$$
\begin{aligned}
Z\left(f, \phi_{1} ; w, s\right)= & Z_{+}\left(f, \phi_{1} ; w, s\right)+Z_{+}^{*}\left(\hat{f}, \hat{\phi}_{1} ; w, \frac{m}{2}+1-w-s\right) \\
& +\frac{|D|^{-1} Z^{*}(0, w)}{w+s-\frac{m}{2}-1} \Sigma^{*}\left(\hat{f} ; w-\frac{m}{2}+1\right) \\
& +\frac{\zeta(w-m+1)}{s-1} \sum_{\epsilon} \Phi_{\epsilon}(f ; w-m, 0) \\
& -\frac{Z(0, w)}{s} \Sigma\left(f ; w-\frac{m}{2}+1\right) \\
& -\frac{\zeta(w-m+1)}{w+s-\frac{m}{2}} \sum_{\eta} \Phi_{\eta}^{*}(\hat{f} ; w-m, 0), \\
Z^{*}\left(f, \hat{\phi}_{1} ; w, s\right)= & Z_{+}^{*}\left(f, \hat{\phi}_{1} ; w, s\right)+Z_{+}\left(\hat{f}, \phi_{1} ; w, \frac{m}{2}+1-w-s\right) \\
& +\frac{Z(0, w)}{w+s-\frac{m}{2}-1} \Sigma\left(\hat{f} ; w-\frac{m}{2}+1\right) \\
& +\frac{\zeta(w-m+1)}{s-1} \sum_{\eta} \Phi_{\eta}^{*}(f ; w-m, 0) \\
& -\frac{|D|^{-1} Z^{*}(0, w)}{s} \Sigma^{*}\left(f ; w-\frac{m}{2}+1\right) \\
& -\frac{\zeta(w-m+1)}{w+s-\frac{m}{2}} \sum_{\epsilon} \Phi_{\epsilon}(\hat{f} ; w-m, 0) .
\end{aligned}
$$

(2) Let $\psi$ be a Dirichlet character whose conductor $r$ is an odd prime not dividing $2|D|$. Then we have

$$
\begin{aligned}
Z\left(f, \phi_{\psi} ; w, s\right)= & Z_{+}\left(f, \phi_{\psi} ; w, s\right)+Z_{+}^{*}\left(\hat{f}, \hat{\phi}_{\psi} ; w, \frac{m}{2}+1-w-s\right) \\
& +\frac{\hat{\phi}_{\psi}(0)|D|^{-1} Z^{*}(0, w)}{w+s-\frac{m}{2}-1} \Sigma^{*}\left(\hat{f} ; w-\frac{m}{2}+1\right) \\
& +\frac{\hat{\phi}_{\psi}(0) r^{m-w} \zeta(w-m+1)}{s-1} \sum_{\epsilon} \Phi_{\epsilon}(f ; w-m, 0), \\
Z^{*}\left(f, \hat{\phi}_{\psi} ; w, s\right)= & Z_{+}^{*}\left(f, \hat{\phi}_{\psi} ; w, s\right)+Z_{+}\left(\hat{f}, \phi_{\psi} ; w, \frac{m}{2}+1-w-s\right) \\
& -\frac{\hat{\phi}_{\psi}(0)|D|^{-1} Z^{*}(0, w)}{s} \Sigma^{*}\left(f ; w-\frac{m}{2}+1\right) \\
& -\frac{\hat{\phi}_{\psi}(0) r^{m-w} \zeta(w-m+1)}{w+s-\frac{m}{2}} \sum_{\epsilon} \Phi_{\epsilon}(\hat{f} ; w-m, 0) .
\end{aligned}
$$


Proof. From the Poisson summation formula, it follows that

$$
\sum_{x \in V_{\mathbb{Q}}} \phi(x) f(x)=\sum_{x \in V_{\mathbb{Q}}} \hat{\phi}(x) \hat{f}(x) .
$$

Hence we have

$$
Z(f, \phi ; w, s)=Z_{+}(f, \phi ; w, s)+Z_{+}^{*}\left(\hat{f}, \hat{\phi} ; w, \frac{m}{2}+1-w-s\right)+\Sigma
$$

where

$$
\begin{aligned}
\Sigma & =\int_{G_{\mathbb{R}} / \Gamma, \chi(p, t) \leq 1} \chi_{1}(p, t)^{w} \chi(p, t)^{s} \\
& \times\left(\chi(p, t)^{-m / 2-1} \sum_{y \in S_{\mathbb{Q}}^{*} \cap V_{\mathbb{Q}}} \hat{\phi}(y) \hat{f}\left(\rho^{*}(g) y\right)-\sum_{x \in S_{\mathbb{Q} \cap V_{\mathbb{Q}}}} \phi(x) f(\rho(g) x)\right) d_{r} g .
\end{aligned}
$$

Since the functions $\phi$ and $\hat{\phi}$ are $\Gamma$-invariants, we see that

$$
\begin{aligned}
\sum_{y \in S_{\mathbb{Q}}^{*} \cap V_{\mathbb{Q}}} \hat{\phi}(y) \hat{f}\left(\rho^{*}(g) y\right) & =\sum_{y \in \Gamma \backslash S_{\mathbb{Q}}^{*} \cap V_{\mathbb{Q}}} \hat{\phi}(y) \sum_{\gamma \in \Gamma / \Gamma_{y}} \hat{f}\left(\rho^{*}(g \gamma) y\right), \\
\sum_{x \in S_{\mathbb{Q}} \cap V_{\mathbb{Q}}} \phi(x) f(\rho(g) x) & =\sum_{x \in \Gamma \backslash S_{\mathbb{Q}} \cap V_{\mathbb{Q}}} \phi(x) \sum_{\gamma \in \Gamma / \Gamma_{x}} f(\rho(g \gamma) x),
\end{aligned}
$$

where $\Gamma_{x}$ denotes the stabilizer of $x$ in $\Gamma$.

We consider the case $\phi=\phi_{1}$. We call two points $x, y$ of $V_{\mathbb{Z}} \rho(\Gamma)$ equivalent (resp. $\rho^{*}(\Gamma)$-equivalent) if they lie in the same $\rho(\Gamma)$-orbit (resp. $\rho^{*}(\Gamma)$-orbit) in $V_{\mathbb{Z}}$. Then it is easy to see that a complete set of representatives of $\rho(\Gamma)$-equivalence classes in $S_{\mathbb{Q}} \cap V_{\mathbb{Z}}$ is given by the union of the following subsets of $V_{\mathbb{Z}}$ :

$$
\begin{aligned}
& L_{1}=\left\{\left(\begin{array}{c}
x_{0} \\
v \\
x_{m+1}
\end{array}\right) \in V_{\mathbb{Z}} \mid x_{m+1} \neq 0, v \bmod x_{m+1} \mathbb{Z}^{m}, Q\left(\begin{array}{c}
x_{0} \\
v \\
x_{m+1}
\end{array}\right)=0\right\}, \\
& L_{2}=\left\{\left(\begin{array}{c}
x_{0} \\
v \\
0
\end{array}\right) \in V_{\mathbb{Z}} \mid v \neq 0, x_{0} \bmod 2 \alpha_{v}\right\}, \\
& L_{3}=\left\{\left(\begin{array}{c}
x_{0} \\
0 \\
0
\end{array}\right) \mid x_{0} \in \mathbb{Z}\right\},
\end{aligned}
$$


where $\alpha_{v}$ is a positive integer such that $v^{\prime}:=\alpha_{v}^{-1} A v$ is a primitive vector. Recall that an integral vector $v={ }^{t}\left(v_{0}, v_{1}, \ldots, v_{m+1}\right)$ is said to primitive if the greatest common divisor $\left(v_{0}, v_{1}, \ldots, v_{m+1}\right)$ equals 1 . It is easy to see that $\Gamma_{x}=\{1\}$ for $x \in L_{1}, \Gamma_{x}=\Gamma$ for $x \in L_{3}$ and

$$
\Gamma_{x}=\left\{\left(\begin{array}{ccc}
1 & -2{ }^{t} u A & -A[u] \\
0 & 1 & u \\
0 & 0 & 1
\end{array}\right)|u \in\langle v\rangle\rangle_{A}^{\perp} \cap \mathbb{Z}^{m}\right\} \text { for } x=\left(\begin{array}{c}
x_{0} \\
v \\
0
\end{array}\right) \in L_{2}
$$

where

$$
\langle v\rangle_{A}^{\perp}=\left\{\left.u \in \mathbb{R}^{m}\right|^{t} u A v=0\right\}
$$

Similarly, the union of the following subsets forms a complete set of representatives of $\rho^{*}(\Gamma)$-equivalence classes in $S_{\mathbb{Q}}^{*} \cap V_{\mathbb{Z}}$ :

$$
\begin{aligned}
& L_{1}^{*}=\left\{\left(\begin{array}{c}
y_{0} \\
v^{*} \\
y_{m+1}
\end{array}\right) \in V_{\mathbb{Z}} \mid y_{0} \neq 0, v^{*} \bmod y_{0} \mathbb{Z}^{m}, Q^{*}\left(\begin{array}{c}
y_{0} \\
v^{*} \\
y_{m+1}
\end{array}\right)=0\right\}, \\
& L_{2}^{*}=\left\{\left(\begin{array}{c}
0 \\
v^{*} \\
y_{m+1}
\end{array}\right) \in V_{\mathbb{Z}} \mid v^{*} \neq 0, y_{m+1} \bmod \alpha_{v^{*}}\right\}, \\
& L_{3}^{*}=\left\{\left(\begin{array}{c}
0 \\
0 \\
y_{m+1}
\end{array}\right) \mid y_{m+1} \in \mathbb{Z}\right\},
\end{aligned}
$$

where $\alpha_{v^{*}}$ is a positive integer such that $\left(v^{*}\right)^{\prime}:=\alpha_{v^{*}}^{-1} v^{*}$ is a primitive vector. We also see that $\Gamma_{y}=\{1\}$ for $y \in L_{1}^{*}, \Gamma_{y}=\Gamma$ for $y \in L_{3}^{*}$ and

$\Gamma_{y}=\left\{\left(\begin{array}{ccc}1 & -2{ }^{t} u A & -A[u] \\ 0 & 1 & u \\ 0 & 0 & 1\end{array}\right) \mid u \in\left\langle v^{*}\right\rangle^{\perp} \cap \mathbb{Z}^{m}\right\}$ for $y=\left(\begin{array}{c}0 \\ v^{*} \\ y_{m+1}\end{array}\right) \in L_{2}^{*}$,

where

$$
\left\langle v^{*}\right\rangle^{\perp}=\left\{\left.u \in \mathbb{R}^{m}\right|^{t} u v^{*}=0\right\}
$$

Using the identities (3.3), (3.4) and the calculation of $\Gamma_{x}$ and $\Gamma_{y}$, we see 
that the third term $\Sigma$ on the right hand side of (3.2) is equal to

$$
\begin{aligned}
2 \times & \int_{0}^{\infty} \int_{0}^{1} a^{m-w-1} t^{w+2 s-1}\left\{I_{1}^{*}\left(\left(f_{t}\right)^{\wedge}\right)+I_{2}^{*}\left(\left(f_{t}\right)^{\wedge}\right)-I_{1}\left(f_{t}\right)-I_{2}\left(f_{t}\right)\right\} d t d a \\
= & 2 \times \int_{0}^{\infty} \int_{0}^{1} a^{m-w-1} t^{w+2 s-1}\left\{I_{1}^{*}\left(\left(f_{t}\right)^{\wedge}\right)-I_{1}\left(f_{t}\right)\right\} d t d a \\
& +2 \times \int_{0}^{\infty} \int_{0}^{1} a^{m-w-1} t^{w+2 s-1}\left\{I_{2}^{*}\left(\left(f_{t}\right)^{\wedge}\right)-I_{2}\left(f_{t}\right)\right\} d t d a
\end{aligned}
$$

where

$$
\begin{aligned}
& I_{1}(f)=\sum_{x \in L_{1}} \int_{\mathbb{R}^{m}} f\left(\begin{array}{c}
a x_{0}-2 a^{t} u A v-a x_{m+1} A[u] \\
v+x_{m+1} u \\
a^{-1} x_{m+1}
\end{array}\right) d u, \\
& I_{2}(f)=\sum_{x \in L_{2}} \int_{\mathbb{R}^{m} /\left(\langle v\rangle{ }_{A}^{\perp} \cap \mathbb{Z}^{m}\right)} f\left(\begin{array}{c}
a x_{0}-2 a^{t} u A v \\
v \\
0
\end{array}\right) d u+\sum_{x \in L_{3}} f\left(\begin{array}{c}
a x_{0} \\
0 \\
0
\end{array}\right), \\
& I_{1}^{*}(f)=\sum_{y \in L_{1}^{*}} \int_{\mathbb{R}^{m}} f\left(\begin{array}{c}
a^{-1} y_{0} \\
2 y_{0} A u+v^{*} \\
-a y_{0} A[u]-a^{t} u v^{*}+a y_{m+1}
\end{array}\right) d u, \\
& I_{2}^{*}(f)=\sum_{y \in L_{2}^{*}} \int_{\mathbb{R}^{m} /\left(\left\langle v^{*}\right\rangle^{\perp} \cap \mathbb{Z}^{m}\right)} f\left(\begin{array}{c}
0 \\
v^{*} \\
-a^{t} u v^{*}+a y_{m+1}
\end{array}\right) d u+\sum_{y \in L_{3}^{*}} f\left(\begin{array}{c}
0 \\
0 \\
a y_{m+1}
\end{array}\right)
\end{aligned}
$$

and $f_{t}(x)=f(t x)$. We can easily calculate the first integral on the right hand side of $(3.5)$ :

$$
\begin{aligned}
& 2 \times \int_{0}^{\infty} \int_{0}^{1} a^{m-w-1} t^{w+2 s-1}\left(I_{1}^{*}\left(\left(f_{t}\right)^{\wedge}\right)-I_{1}\left(f_{t}\right)\right) d a d t \\
&=|D|^{-1}\left(w+s-\frac{m}{2}-1\right)^{-1}\left(\sum_{y \in L_{1}^{*}}\left|y_{0}\right|^{-w}\right) \Sigma^{*}\left(\hat{f} ; w-\frac{m}{2}+1\right) \\
& \quad-s^{-1}\left(\sum_{x \in L_{1}}\left|x_{m+1}\right|^{-w}\right) \Sigma\left(f ; w-\frac{m}{2}+1\right) .
\end{aligned}
$$

Next we consider the second integral involving $I_{2}^{*}(\hat{f})-I_{2}(f)$. Put $A v=\alpha_{v} v^{\prime}$, where the vector $v^{\prime}$ is a primitive vector, then we choose a set of vectors $\left\{v_{1}, \ldots, v_{m-1}\right\}$ such that $U=\left(v^{\prime}, v_{1}, \ldots, v_{m-1}\right) \in G L(m, \mathbb{Z})$. We take a 
basis of $V_{\mathbb{R}}$ of the form $\left\{f_{1}, f_{2}, \ldots, f_{m}\right\}$ for ${ }^{t} U^{-1}=\left(f_{1}, f_{2}, \ldots, f_{m}\right)$. Denote a vector $u$ of $V_{\mathbb{R}}$ by $u=c_{1} f_{1}+c_{2} f_{2}+\cdots+c_{m} f_{m}$, then ${ }^{t} u v^{\prime}=0$ if and only if $c_{1}=0$. Hence we see that the subspace $\left\langle v^{\prime}\right\rangle^{\perp}$ is spanned by the vectors $\left\{f_{2}, \ldots, f_{m}\right\}$. Since $d u=d c_{1} \cdots d c_{m}$, we see that

$$
\begin{aligned}
I_{2}^{*}(\hat{f})-I_{2}(f)= & a^{-1} \sum_{v^{*} \in \mathbb{Z}^{m}-\{0\}} \int_{\mathbb{R}} \hat{f}\left(\begin{array}{c}
0 \\
v^{*} \\
c
\end{array}\right) d c+\sum_{y \in \mathbb{Z}} \hat{f}\left(\begin{array}{c}
0 \\
0 \\
a y
\end{array}\right) \\
& -a^{-1} \sum_{v \in \mathbb{Z}^{m}-\{0\}} \int_{\mathbb{R}} f\left(\begin{array}{c}
c \\
v \\
0
\end{array}\right) d c-\sum_{x \in \mathbb{Z}} f\left(\begin{array}{c}
a x \\
0 \\
0
\end{array}\right) .
\end{aligned}
$$

We can see easily that

$$
\begin{aligned}
\sum_{v^{*} \in \mathbb{Z}^{m}-\{0\}} \int_{\mathbb{R}} \hat{f}\left(\begin{array}{c}
0 \\
v^{*} \\
c
\end{array}\right) d c & =\sum_{v^{*} \in \mathbb{Z}^{m}} \int_{\mathbb{R}} \hat{f}\left(\begin{array}{c}
0 \\
v^{*} \\
c
\end{array}\right) d c-\int_{\mathbb{R}} \hat{f}\left(\begin{array}{c}
0 \\
0 \\
c
\end{array}\right) d c \\
& =\sum_{v^{*} \in \mathbb{Z}^{m}} \int_{\mathbb{R}} \hat{f}\left(\begin{array}{c}
0 \\
v^{*} \\
c
\end{array}\right) d c-\int_{\mathbb{R}^{m+1}} f\left(\begin{array}{l}
x \\
u \\
0
\end{array}\right) d x d u
\end{aligned}
$$

From the Poisson summation formula, we have

$$
\begin{aligned}
\sum_{v^{*} \in \mathbb{Z}^{m}} \int_{\mathbb{R}} \hat{f}\left(\begin{array}{c}
0 \\
v^{*} \\
c
\end{array}\right) d c & =\sum_{v \in \mathbb{Z}^{m}} \int_{\mathbb{R}} f\left(\begin{array}{c}
c \\
v \\
0
\end{array}\right) d c \\
& =\sum_{v \in \mathbb{Z}^{m}-\{0\}} \int_{\mathbb{R}} f\left(\begin{array}{c}
c \\
v \\
0
\end{array}\right) d c+\int_{\mathbb{R}} f\left(\begin{array}{c}
c \\
0 \\
0
\end{array}\right) d c \\
& =\sum_{v \in \mathbb{Z}^{m}-\{0\}} \int_{\mathbb{R}} f\left(\begin{array}{c}
c \\
v \\
0
\end{array}\right) d c+\int_{\mathbb{R}^{m+1}} \hat{f}\left(\begin{array}{l}
0 \\
u \\
x
\end{array}\right) d x d u \\
\sum_{y \in \mathbb{Z}} \hat{f}\left(\begin{array}{c}
0 \\
0 \\
a y
\end{array}\right) & =a^{-1} \sum_{l \in \mathbb{Z}} \int_{\mathbb{R}^{m+1}} f\left(\begin{array}{c}
x \\
u \\
a^{-1} l
\end{array}\right) d x d u \\
\sum_{x \in \mathbb{Z}} f\left(\begin{array}{c}
a x \\
0 \\
0
\end{array}\right) & =a^{-1} \sum_{l \in \mathbb{Z}} \int_{\mathbb{R}^{m+1}} \hat{f}\left(\begin{array}{c}
a^{-1} l \\
u \\
x
\end{array}\right) d x d u
\end{aligned}
$$


Hence we obtain that

$$
\begin{aligned}
I_{2}^{*}(\hat{f})-I_{2}(f)= & a^{-1} \sum_{l \in \mathbb{Z}-\{0\}} \int_{\mathbb{R}^{m+1}} f\left(\begin{array}{c}
x \\
u \\
a^{-1} l
\end{array}\right) d x d u \\
& -a^{-1} \sum_{l \in \mathbb{Z}-\{0\}} \int_{\mathbb{R}^{m+1}} \hat{f}\left(\begin{array}{c}
a^{-1} l \\
u \\
x
\end{array}\right) d x d u .
\end{aligned}
$$

Thus we have

$$
\begin{aligned}
2 \times & \int_{0}^{\infty} \int_{0}^{1} a^{m-w-2} t^{w+2 s-1}\left(I_{2}^{*}\left(\left(f_{t}\right)^{\wedge}\right)-I_{2}\left(f_{t}\right)\right) d t d a \\
= & (s-1)^{-1} \zeta(w-m+1)\left(\Phi_{+}(f ; w-m, 0)+\Phi_{-}(f ; w-m, 0)\right) \\
& -\left(w+s-\frac{m}{2}\right)^{-1} \zeta(w-m+1)\left(\Phi_{+}^{*}(\hat{f} ; w-m, 0)+\Phi_{-}^{*}(\hat{f} ; w-m, 0)\right) .
\end{aligned}
$$

This proves the assertion for $\phi_{1}$. We note that $\phi_{\psi}(x)=0$ if $Q(x)=0$. Our assertion for $\phi_{\psi}$ can be proved in the same way as in the case of $\phi_{1}$.

Corollary 3.7. For $\phi=\phi_{1}$ or $\phi_{\psi}, Z(f, \phi ; w, s)$ and $Z^{*}(\hat{f}, \hat{\phi} ; w, s)$ have analytic continuations to meromorphic functions in the domain $\left\{(w, s) \in \mathbb{C}^{2} \mid \operatorname{Re}(w)>m\right\}$. Moreover they satisfy the functional equation:

$$
Z(f, \phi ; w, s)=Z^{*}\left(\hat{f}, \hat{\phi} ; w, \frac{m}{2}+1-w-s\right) .
$$

Using Proposition 2.2, we obtain the following corollary.

Corollary 3.8. If $f \in C_{0}^{\infty}\left(V_{\epsilon}\right)$, then the following identities hold:

(1) $Z\left(f, \phi_{1} ; w, s\right)=Z_{+}\left(f, \phi_{1} ; w, s\right)+Z_{+}^{*}\left(\hat{f}, \hat{\phi}_{1} ; w, \frac{m}{2}+1-w-s\right)$

$$
\begin{aligned}
& +\frac{2|D|^{-1 / 2}(2 \pi)^{m / 2-w} \Gamma\left(w-\frac{m}{2}\right) Z^{*}(0, w)}{w+s-\frac{m}{2}-1} \\
& \quad \times \cos \frac{\pi(p-q+\epsilon(m-2 w))}{4} \Phi_{\epsilon}\left(f ; w-m, \frac{m}{2}-w\right) \\
& +\frac{\zeta(w-m+1)}{s-1} \Phi_{\epsilon}(f ; w-m, 0) .
\end{aligned}
$$


(2) $Z\left(f, \phi_{\psi} ; w, s\right)=Z_{+}\left(f, \phi_{\psi} ; w, s\right)+Z_{+}^{*}\left(\hat{f}, \hat{\phi}_{\psi} ; w, \frac{m}{2}+1-w-s\right)$

$$
\begin{aligned}
& +\frac{\hat{\phi}_{\psi}(0)|D|^{-1 / 2}(2 \pi)^{m / 2-w} \Gamma\left(w-\frac{m}{2}\right) Z^{*}(0, w)}{w+s-\frac{m}{2}-1} \\
& \quad \times \cos \frac{\pi(p-q+\epsilon(m-2 w))}{4} \Phi_{\epsilon}\left(f ; w-m, \frac{m}{2}-w\right) \\
& +\frac{\hat{\phi}_{\psi}(0) r^{m-w} \zeta(w-m+1)}{s-1} \Phi_{\epsilon}(f ; w-m, 0) .
\end{aligned}
$$

Proof. It is sufficient to prove only $\sum_{\eta} \Phi_{\eta}^{*}(\hat{f} ; w-m, 0)=0$. If the support of $f$ is contained in $V_{+}$, the function $\Phi_{-}(f ; w, s)$ vanishes identically. From Theorem 2.1, we have

$$
\begin{aligned}
& \cos \frac{\pi(w-p)}{2} \Phi_{+}^{*}\left(\hat{f} ; w-m, \frac{m}{2}-w-s\right) \\
&+\cos \frac{\pi(w+2 s-q)}{2} \Phi_{-}^{*}\left(\hat{f} ; w-m, \frac{m}{2}-w-s\right)=0 .
\end{aligned}
$$

In the same manner, we have

$$
\begin{aligned}
& \cos \frac{\pi(w+2 s-p)}{2} \Phi_{+}^{*}\left(\hat{f} ; w-m, \frac{m}{2}-w-s\right) \\
& +\cos \frac{\pi(w-q)}{2} \Phi_{-}^{*}\left(\hat{f} ; w-m, \frac{m}{2}-w-s\right)=0
\end{aligned}
$$

if the support of $f$ is contained in $V_{-}$. When $s$ tends to $m / 2-w$ in $(3.6)$ and (3.7), we have

$$
\sum_{\eta} \Phi_{\eta}^{*}(\hat{f} ; w-m, 0)=0
$$

\section{§4. Main results}

First, we give the functional equation satisfied by $\zeta_{\epsilon}(\phi ; w, s)$ and $\zeta_{\eta}^{*}(\hat{\phi} ; w, s)$ for the cases $\phi=\phi_{1}$ and $\phi_{\psi}$ and calculate their poles and residues.

THEOREM 4.1. The zeta functions $\zeta_{\epsilon}(\phi ; w, s)$ and $\zeta_{\eta}^{*}(\hat{\phi} ; w, s)\left(\phi=\phi_{1}\right.$ or $\left.\phi_{\psi}\right)$ have analytic continuations to meromorphic functions in the domain

$$
\mathfrak{D}=\left\{(w, s) \in \mathbb{C}^{2} \mid \operatorname{Re}(w)>m\right\}
$$

with the following properties. 
(1) They satisfy the following functional equations for $\phi=\phi_{1}$ or $\phi_{\psi}$ :

$$
\left(\begin{array}{c}
\zeta_{+}^{*} \\
\zeta_{-}^{*}
\end{array}\right)\left(\hat{\phi} ; w, \frac{m}{2}+1-w-s\right)={ }^{t} \gamma(w, s)\left(\begin{array}{c}
\zeta_{+} \\
\zeta_{-}
\end{array}\right)(\phi ; w, s),
$$

where

$$
\begin{gathered}
\gamma(w, s)=2|D|^{-1 / 2}(2 \pi)^{m / 2-w-2 s} \Gamma(s) \Gamma\left(w+s-\frac{m}{2}\right) \\
\times\left(\begin{array}{cc}
\cos \frac{\pi(w+2 s-p)}{2} & \cos \frac{\pi(w-q)}{2} \\
\cos \frac{\pi(w-p)}{2} & \cos \frac{\pi(w+2 s-q)}{2}
\end{array}\right) .
\end{gathered}
$$

(2) Fix a complex number $w$ with $\operatorname{Re}(w)>m$.

(i) The function $(s-1)(s+w-m / 2-1) \zeta_{\epsilon}\left(\phi_{1} ; w, s\right)$ is an entire function of $s$. The residues of $\zeta_{\epsilon}\left(\phi_{1} ; w, s\right)$ are given by

$$
\begin{aligned}
& \operatorname{Res}_{s=1} \zeta_{\epsilon}\left(\phi_{1} ; w, s\right)= \zeta(w-m+1) \\
& \operatorname{Res}_{s=m / 2+1-w} \zeta_{\epsilon}\left(\phi_{1} ; w, s\right)=\frac{2 \Gamma\left(w-\frac{m}{2}\right) Z^{*}(0, w)}{|D|^{1 / 2}(2 \pi)^{w-m / 2}} \\
& \\
& \times \cos \frac{\pi(p-q+\epsilon(m-2 w))}{4} .
\end{aligned}
$$

(ii) The function $(s-1)(s+w-m / 2-1) \zeta_{\eta}^{*}\left(\hat{\phi}_{1} ; w, s\right)$ is an entire function of $s$. The residues of $\zeta_{\eta}^{*}\left(\hat{\phi}_{1} ; w, s\right)$ are given by

$$
\begin{aligned}
\operatorname{Res}_{s=1} \zeta_{\eta}^{*}\left(\hat{\phi}_{1} ; w, s\right)= & \zeta(w-m+1), \\
\operatorname{Res}_{s=m / 2+1-w} \zeta_{\eta}^{*}\left(\hat{\phi}_{1} ; w, s\right)= & \frac{2 \Gamma\left(w-\frac{m}{2}\right) Z(0, w)}{|D|^{1 / 2}(2 \pi)^{w-m / 2}} \\
& \times \cos \frac{\pi(q-p+\eta(m-2 w))}{4} .
\end{aligned}
$$

(iii) If $m$ is even or $\psi \neq\left(\frac{*}{r}\right)$, then the functions $\zeta_{\epsilon}\left(\phi_{\psi} ; w, s\right)$ and $\zeta_{\eta}^{*}\left(\hat{\phi}_{\psi} ; w, s\right)$ are entire functions of $s$.

(iv) If $m$ is odd and $\psi=\left(\frac{*}{r}\right)$, then the functions $(s-1)(s+w-m / 2-$ $1) \zeta_{\epsilon}\left(\phi_{\left(\frac{*}{r}\right)} ; w, s\right)$ and $\zeta_{\eta}^{*}\left(\hat{\phi}_{\left(\frac{*}{r}\right)} ; w, s\right)$ are entire functions of $s$. The resi- 
dues of $\zeta_{\epsilon}\left(\phi_{\left(\frac{*}{r}\right)} ; w, s\right)$ are given by

$$
\begin{aligned}
& \operatorname{Res}_{s=1} \zeta_{\epsilon}\left(\phi_{\left(\frac{*}{r}\right)} ; w, s\right)=r^{m / 2-w-1} \alpha \zeta(w-m+1) \\
& \operatorname{Res}_{s=m / 2+1-w} \zeta_{\epsilon}\left(\phi_{\left(\frac{*}{r}\right)} ; w, s\right)=\frac{2 \alpha \Gamma\left(w-\frac{m}{2}\right) Z(0, w)}{|D|^{1 / 2}(2 \pi)^{w-m / 2}} \\
& \times \cos \frac{\pi(q-p+\eta(m-2 w))}{4},
\end{aligned}
$$

where $\alpha=(r-1) \times \epsilon_{r}^{m+1}\left(\frac{2 D}{r}\right) r^{-1 / 2}$.

Proof. Our assertions for the analytic continuations and functional equations follow immediately from Proposition 2.1, Proposition 3.5 and Corollary 3.7. Now we compute the residues. We have the following identity for any $f \in C_{0}^{\infty}\left(V_{\epsilon}\right)$ from Corollary $3.8(1)$ :

$$
\begin{aligned}
\Phi_{\epsilon}(f ; w-m, s-1) \zeta_{\epsilon}\left(\phi_{1} ; w, s\right) \\
=Z_{+}\left(f, \phi_{1} ; w, s\right)+Z_{+}^{*}\left(\hat{f}, \hat{\phi}_{1} ; w, \frac{m}{2}+1-w-s\right) \\
+\left(w+s-\frac{m}{2}-1\right)^{-1} 2|D|^{-1 / 2}(2 \pi)^{m / 2-w} \Gamma\left(w-\frac{m}{2}\right) \\
\quad \times \cos \frac{\pi(p-q+\epsilon(m-2 w))}{4} Z^{*}(0 ; w) \Phi_{\epsilon}\left(f ; w-m, \frac{m}{2}-w\right) \\
\quad+(s-1)^{-1} \zeta(w-m+1) \Phi_{\epsilon}(f ; w-m, 0) .
\end{aligned}
$$

Since there exists an $f \in C_{0}^{\infty}\left(V_{\epsilon}\right)$ such that $\Phi_{\epsilon}(f ; w-m, 0) \neq 0$, we see that the function $\zeta_{\epsilon}\left(\phi_{1} ; w, s\right)$ has a pole at $s=1$ and the residue is $\zeta(w-m+1)$. In the same manner as above, we obtain the assertion for the residue of $\zeta_{\epsilon}\left(\phi_{1} ; w, s\right)$ at $s=m / 2+1-w$. Our assertion for $\zeta_{\eta}^{*}\left(\hat{\phi}_{1} ; w, s\right)$ can be proved in the same way as in the case of $\zeta_{\epsilon}\left(\phi_{1} ; w, s\right)$. Using Corollary $3.8(2)$, we can prove our assertion for $\zeta_{\epsilon}\left(\phi_{\psi} ; w, s\right)$ and $\zeta_{\eta}^{*}\left(\phi_{\psi} ; w, s\right)$ in the same way.

Moreover using Lemma 3.3, we can extend $\zeta_{\epsilon}(\phi ; w, s)$ in $\mathbb{C}^{2}$.

Corollary 4.2. For $\phi=\phi_{1}$ or $\phi_{\psi}, \zeta_{\epsilon}(\phi ; w, s)$ has an analytic continuation to a meromorphic function in $\mathbb{C}^{2}$.

Proof. Define

$$
g(w):=\zeta(w-m+1)^{-1} L\left(w-\frac{m}{2}+1,\left(\frac{(-1)^{m / 2+1} 2 D}{*}\right)\right)
$$


if $m$ is even and

$$
g(w):=\left(w-\frac{m+1}{2}\right) \zeta(w-m+1)^{-1} \zeta(2 w-m+1) \prod_{p \mid D}\left(1-p^{m-1-2 w}\right)
$$

if $m$ is odd and put

$$
G(w, s):=(s-1)\left(s+w-\frac{m}{2}\right) g(w) \zeta_{\epsilon}\left(\phi_{1} ; w, s\right) .
$$

From Lemma 3.3 and Lemma 3.4, we have

$$
G(w, s)=(s-1)\left(s+w-\frac{m}{2}\right) \sum_{n=1}^{\infty} F(\epsilon n, w) n^{-s}
$$

From Theorem 4.1 and the estimate of $F(\epsilon n, w)$ in Lemma 3.3, there exists a positive number $c$ for any $\sigma_{1} \in \mathbb{R}$ such that $G(w, s)$ is holomorphic in the domain

$$
\left\{(w, s) \in \mathbb{C}^{2} \mid \operatorname{Re}(w)>m\right\} \cup\left\{(w, s) \in \mathbb{C}^{2} \mid \operatorname{Re}(w)>\sigma_{1}, \operatorname{Re}(s)>1+c\right\} .
$$

Since the convex hull of this domain coincides with $\left\{(w, s) \in \mathbb{C}^{2} \mid \operatorname{Re}(w)>\right.$ $\left.\sigma_{1}\right\}$ and $\sigma_{1}$ is arbitrary, the assertion for $\phi=\phi_{1}$ follows from Theorem 2.5.10 in [2]. The proof for $\phi=\phi_{\psi}$ is similar to the case for $\phi=\phi_{1}$.

Let $k$ be a positive integer greater than $(m+\epsilon(q-p)-2) / 4$ and put $w=c(\epsilon, k)+m / 2$ in the above proposition with the constant $c(\epsilon, k)=$ $2 k+1+\epsilon(p-q) / 2,(c(\epsilon, k)+m / 2>m$ by the assumption for $k)$. Then the $\gamma$-matrix $\gamma(w, s)$ in Theorem 4.1 becomes an upper or lower triangular matrix. Hence we have the functional equations below:

$$
\begin{aligned}
& |D|^{1 / 2}(2 \pi)^{-(c(\epsilon, k)+1-s)} \Gamma(c(\epsilon, k)+1-s) \zeta_{\epsilon}^{*}\left(\hat{\phi} ; c(\epsilon, k)+\frac{m}{2}, 1-s\right) \\
& =(-1)^{k+1}(2 \pi)^{-s} \Gamma(s) \zeta_{\epsilon}\left(\phi ; c(\epsilon, k)+\frac{m}{2}, s-c(\epsilon, k)\right) .
\end{aligned}
$$

This functional equation suggests that $\zeta_{\epsilon}$ and $\zeta_{\epsilon}^{*}$ are related to modular forms. 
Now we introduce Dirichlet series $L_{\epsilon}(k ; s)$ and $L_{\epsilon}^{*}(k ; s)$, which will turn out to be the Mellin transforms of modular forms, as follows:

$$
\begin{aligned}
L_{\epsilon}(k ; s) & :=\zeta_{\epsilon}\left(\phi_{1} ; c(\epsilon, k)+\frac{m}{2}, s-c(\epsilon, k)\right)=\sum_{n=1}^{\infty} a_{\epsilon}(k ; n) n^{-s}, \\
L_{\epsilon}^{*}(k ; s) & :=(-1)^{k+1}|D|^{1 / 2} \tilde{D}^{c(\epsilon, k) / 2+1 / 2-s} \zeta_{\epsilon}^{*}\left(\hat{\phi}_{1} ; c(\epsilon, k)+\frac{m}{2}, s-c(\epsilon, k)\right) \\
& =\sum_{n=1}^{\infty} b_{\epsilon}(k ; n) n^{-s},
\end{aligned}
$$

where

$$
\tilde{D}= \begin{cases}|D| & \text { if } m \text { is even and }|D| \not \equiv 2(\bmod 4), \\ 4|D| & \text { if } m \text { is even and }|D| \equiv 2(\bmod 4), \\ 2|D| & \text { if } m \text { is odd }\end{cases}
$$

From Lemma 3.4, we see that

$$
\begin{aligned}
& a_{\epsilon}(k ; n)=n^{c(\epsilon, k)} Z\left(\epsilon n, c(\epsilon, k)+\frac{m}{2}\right), \\
& b_{\epsilon}(k ; n)=(-1)^{k+1} \tilde{D}^{1-c(\epsilon, k) / 2} n^{c(\epsilon, k)} \\
& \quad \times \begin{cases}\delta(n) Z^{*}(\epsilon n / 4, c(\epsilon, k)+m / 2) & \text { if } m \text { is even and }|D| \equiv 2(\bmod 4), \\
Z^{*}(\epsilon n, c(\epsilon, k)+m / 2) & \text { otherwise. }\end{cases}
\end{aligned}
$$

For a positive integer $N$, we set

$$
\begin{aligned}
& \Lambda_{N}\left(s ; k, L_{\epsilon}\right)=\left(\frac{2 \pi}{\sqrt{N}}\right)^{-s} \Gamma(s) L_{\epsilon}(k ; s), \\
& \Lambda_{N}\left(s ; k, L_{\epsilon}^{*}\right)=\left(\frac{2 \pi}{\sqrt{N}}\right)^{-s} \Gamma(s) L_{\epsilon}^{*}(k ; s) .
\end{aligned}
$$

Proposition 4.3. The functions $\Lambda_{\tilde{D}}\left(s ; k, L_{\epsilon}\right)$ and $\Lambda_{\tilde{D}}\left(s ; k, L_{\epsilon}^{*}\right)$ are meromorphic in the complex plane and satisfy the functional equation

$$
\Lambda_{\tilde{D}}\left(1+c(\epsilon, k)-s ; k, L_{\epsilon}^{*}\right)=\Lambda_{\tilde{D}}\left(s ; k, L_{\epsilon}\right)
$$

and the function

$$
\Lambda_{\tilde{D}}\left(s ; k, L_{\epsilon}\right)+\frac{a_{\epsilon}(k ; 0)}{s}+\frac{b_{\epsilon}(k ; 0)}{c(\epsilon, k)+1-s}
$$


is holomorphic on the whole s-plane, where

$$
\begin{aligned}
& a_{\epsilon}(k ; 0)=\frac{(-1)^{k+1}|D|^{1 / 2} \Gamma(c(\epsilon, k)+1) \zeta\left(c(\epsilon, k)-\frac{m}{2}+1\right)}{(2 \pi)^{c(\epsilon, k)+1}}, \\
& b_{\epsilon}(k ; 0)=\frac{\tilde{D}^{1 / 2(c(\epsilon, k)+1)} \Gamma(c(\epsilon, k)+1) \zeta\left(c(\epsilon, k)-\frac{m}{2}+1\right)}{(2 \pi)^{c(\epsilon, k)+1}} .
\end{aligned}
$$

Proof. The identity (4.1) implies the above functional equations. Since $\Gamma(s)$ has poles at $s=0,-1, \ldots$, it follows from Theorem 4.1 that the possible poles of $\Lambda_{\tilde{D}}\left(s ; k, L_{\epsilon}\right)$ are at $s=c(\epsilon, k)+1$ and $s=0,-1, \ldots$ On the other hand, from the expression on the left hand side of (4.3), we see that the possible poles of $\Lambda_{\tilde{D}}\left(s ; k, L_{\epsilon}\right)$ are at $s=0$ and $s=c(\epsilon, k)+1, c(\epsilon, k)+2, \ldots$ Therefore we deduce that the function $\Lambda_{\tilde{D}}\left(s ; k, L_{\epsilon}\right)$ has poles only at $s=0$ and $s=c(\epsilon, k)+1$ and the residues are

$$
\begin{array}{ll}
\frac{(-1)^{k+1}|D|^{1 / 2} \Gamma(c(\epsilon, k)+1) \zeta\left(c(\epsilon, k)-\frac{m}{2}+1\right)}{(2 \pi)^{c(\epsilon, k)+1}} & (s=0), \\
\frac{\tilde{D}^{1 / 2(c(\epsilon, k)+1)} \Gamma(c(\epsilon, k)+1) \zeta\left(c(\epsilon, k)-\frac{m}{2}+1\right)}{(2 \pi)^{c(\epsilon, k)+1}} & (s=c(\epsilon, k)+1) .
\end{array}
$$

Let $r$ be a prime number not dividing $2|D|$. For a primitive Dirichlet character $\psi$ modulo $r$, we define Dirichlet series $L_{\epsilon}(k ; s, \psi)$ and $L_{\epsilon}^{*}(k ; s, \psi)$ as follows:

$$
\begin{aligned}
& L_{\epsilon}(k ; s, \psi):=\sum_{n=1}^{\infty} \psi(n) a_{\epsilon}(k ; n) n^{-s}, \\
& L_{\epsilon}^{*}(k ; s, \psi) \\
& \quad:= \begin{cases}r \sum_{n=1}^{\infty} b_{\epsilon}(k ; r n)(r n)^{-s}-\sum_{n=1}^{\infty} b_{\epsilon}(k ; n) n^{-s} & \text { if } m \text { is odd and } \psi=\left(\frac{*}{r}\right), \\
\sum_{n=1}^{\infty} \psi(n) b_{\epsilon}(k ; n) n^{-s} & \text { otherwise. }\end{cases}
\end{aligned}
$$

Then, by Lemma 3.4 and the definition of $a_{\epsilon}(k ; n)$ and $b_{\epsilon}(k, n)$, we have

$$
L_{\epsilon}(k ; s, \psi)=\psi(\epsilon) \zeta_{\epsilon}\left(\phi_{\psi} ; c(\epsilon, k)+\frac{m}{2}, s-c(\epsilon, k)\right)
$$


We also have

$$
\begin{aligned}
L_{\epsilon}^{*}(k ; s, \bar{\psi})=( & -1)^{k+1}|D|^{1 / 2}\left(r \tilde{D}^{1 / 2}\right)^{c(\epsilon, k)+1-2 s} C_{\psi}^{-1} \\
& \times \zeta_{\epsilon}^{*}\left(\hat{\phi}_{\psi} ; c(\epsilon, k)+\frac{m}{2}, s-c(\epsilon, k)\right) \\
& \times \begin{cases}1 & \text { if } m \text { is odd and } \psi=\left(\frac{*}{r}\right), \\
\bar{\psi}(\epsilon) & \text { otherwise }\end{cases}
\end{aligned}
$$

where

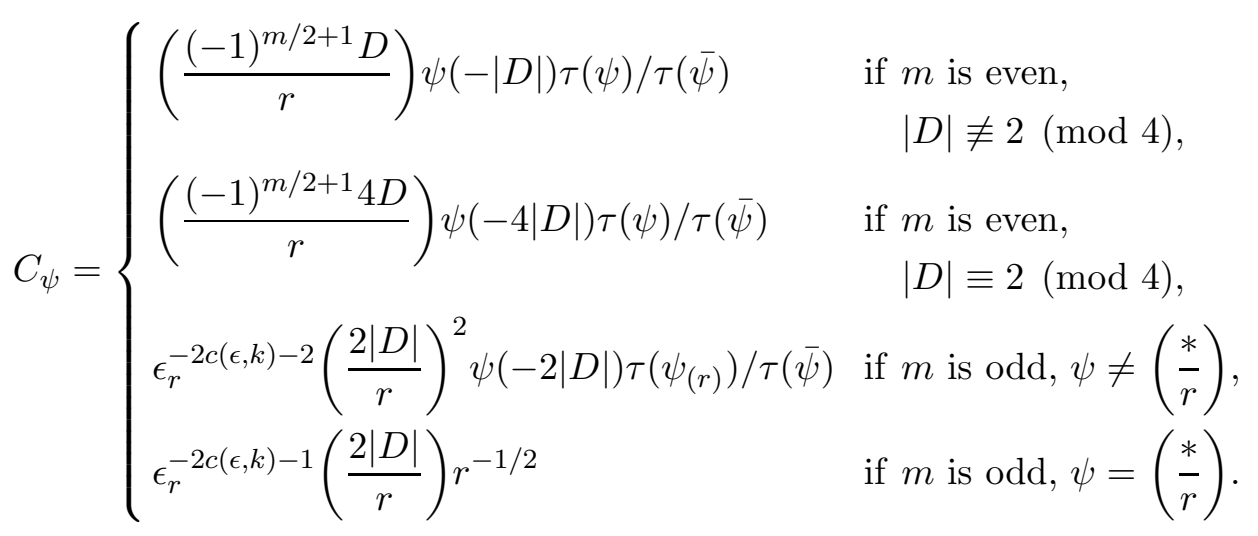

For a positive integer $N$, we set

$$
\begin{aligned}
& \Lambda_{N}\left(s ; k, L_{\epsilon}, \psi\right)=\left(\frac{2 \pi}{\sqrt{N}}\right)^{-s} \Gamma(s) L_{\epsilon}(k ; s, \psi), \\
& \Lambda_{N}\left(s ; k, L_{\epsilon}^{*}, \bar{\psi}\right)=\left(\frac{2 \pi}{\sqrt{N}}\right)^{-s} \Gamma(s) L_{\epsilon}^{*}(k ; s, \bar{\psi}) .
\end{aligned}
$$

The proof of the following proposition is similar to the proof of Proposition 4.3 .

Proposition 4.4. Let $r$ be a prime number not dividing $2|D|$ and $\psi$ a primitive Dirichlet character modulo $r$. Then the functions $\Lambda_{\tilde{D} r^{2}}\left(s ; k, L_{\epsilon}, \psi\right)$ and $\Lambda_{\tilde{D} r^{2}}\left(s ; k, L_{\epsilon}^{*}, \bar{\psi}\right)$ have analytic continuations to meromorphic function of $s$ in $\mathbb{C}$ and satisfy the following functional equation:

$$
\Lambda_{\tilde{D} r^{2}}\left(s ; k, L_{\epsilon}, \psi\right)=C_{\psi} \Lambda_{\tilde{D} r^{2}}\left(c(\epsilon, k)+1-s ; L_{\epsilon}^{*}, \bar{\psi}\right)
$$


The function

$$
\begin{aligned}
& \Lambda_{\tilde{D} r^{2}}\left(s ; k, L_{\epsilon}, \psi\right)+\frac{b_{\epsilon}(k ; 0) r^{-1 / 2}(r-1) \epsilon_{r}^{-1-2 c(\epsilon, k)}\left(\frac{2|D|}{r}\right)}{c(\epsilon, k)+1-s} \\
& \quad \times\left\{\begin{array}{l}
1 \quad \text { if } m \text { is odd and } \psi=\left(\frac{*}{r}\right), \\
0 \quad \text { otherwise }
\end{array}\right.
\end{aligned}
$$

is holomorphic on the whole s-plane.

Let $\mathfrak{H}$ be the upper half complex plane, $\mathfrak{G}_{k}(N, \chi)$ the space of holomorphic modular forms on $\mathfrak{H}$ of the integral weight $k$ and character $\chi$ with respect to $\Gamma_{0}(N)$ and $G_{k}(N, \chi)$ the space of holomorphic modular forms on $\mathfrak{H}$ of the half integral weight $k$ and character $\chi$ with respect to $\Gamma_{0}(N)$, for the definition, see [3] for integral $k$ and [10] for half integral $k$. The following is our main theorem.

THEOREM 4.5. Let $k$ be a positive integer with $c(\epsilon, k)>m / 2$. Let $\left\{a_{\epsilon}(k ; n)\right\}_{n \geq 1}$ and $\left\{b_{\epsilon}(k ; n)\right\}_{n \geq 1}$ be as above and put

$$
\begin{aligned}
& a_{\epsilon}(k ; 0)=\frac{(-1)^{k+1}|D|^{1 / 2} \Gamma(c(\epsilon, k)+1) \zeta\left(c(\epsilon, k)-\frac{m}{2}+1\right)}{(2 \pi)^{c(\epsilon, k)+1}}, \\
& b_{\epsilon}(k ; 0)=\frac{\tilde{D}^{1 / 2(c(\epsilon, k)+1)} \Gamma(c(\epsilon, k)+1) \zeta\left(c(\epsilon, k)-\frac{m}{2}+1\right)}{(2 \pi)^{c(\epsilon, k)+1}} .
\end{aligned}
$$

(1) If $m$ is even, set

$$
\begin{aligned}
& f_{\epsilon}(k ; z)=\sum_{n=0}^{\infty} a_{\epsilon}(k ; n) e(n z), \\
& g_{\epsilon}(k ; z)=\sqrt{-1}^{-c(\epsilon, k)-1} \sum_{n=0}^{\infty} b_{\epsilon}(k ; n) e(n z),
\end{aligned}
$$

Then both $f_{\epsilon}(k ; z)$ and $g_{\epsilon}(k ; z)$ belong to $\mathfrak{G}_{c(\epsilon, k)+1}\left(|D|,\left(\frac{(-1)^{m / 2+1} D}{*}\right)\right)$ or $\mathfrak{G}_{c(\epsilon, k)+1}\left(4|D|,\left(\frac{(-1)^{m / 2+1} 4 D}{*}\right)\right)$ according as $|D| \not \equiv 2 \bmod 4$ or $|D| \equiv 2$ mod 4 respectively. They satisfy the following relation:

$$
g_{\epsilon}(k ; z)=\left(\tilde{D}^{1 / 2} z\right)^{-c(\epsilon, k)-1} f_{\epsilon}\left(k ; \frac{-1}{\tilde{D} z}\right) .
$$


(2) If $m$ is odd, set

$$
\begin{aligned}
& f_{\epsilon}(k ; z)=\sum_{n=0}^{\infty} a_{\epsilon}(k ; n) e(n z), \\
& g_{\epsilon}(k ; z)=\sum_{n=0}^{\infty} b_{\epsilon}(k ; n) e(n z),
\end{aligned}
$$

Then $f_{\epsilon}(k ; z)$ belongs to $G_{c(\epsilon, k)+1}\left(2|D|,\left(\frac{2|D|}{*}\right)\right)$ and $g_{\epsilon}(k ; z)$ belongs to $G_{c(\epsilon, k)+1}\left(2|D|, \mathrm{id}_{2|D|}\right)$. Moreover they satisfy the following relation:

$$
g_{\epsilon}(k ; z)=\left(-\sqrt{-1} \tilde{D}^{1 / 2} z\right)^{-c(\epsilon, k)-1} f_{\epsilon}\left(k ; \frac{-1}{\tilde{D} z}\right) .
$$

To prove this theorem, we apply the converse theorem to the functions $\Lambda_{\tilde{D}}\left(s ; k, L_{\epsilon}\right)$ and $\Lambda_{\tilde{D} r^{2}}\left(s ; k, L_{\epsilon}, \psi\right)$. The converse theorem is proved in [15] for the case of integral weight (see Theorem 4.3.15 in [3]). For the case of half integral weight, Shimura [10, pp. 479-481] described the functional equations of the Dirichlet series given by the Mellin transform of half integral weight modular forms and he pointed out that the functional equations characterize modular forms of half integral weight. The proof of the characterization of modular forms is omitted in [10], since it is almost the same as that in [15].

Proof of Theorem 4.5. Propositions 4.3 and 4.4 show that the functions $\Lambda$ and $\Lambda^{*}$ satisfy the conditions of the converse theorem, except the boundedness of the functions given by (4.2), (4.3). Therefore it suffices to prove the boundedness of the functions (4.2), (4.3) on any vertical strip. To show this, we consider a function $q(t) \in C^{\infty}(\mathbb{R})$, satisfying the following two conditions. The existence of such a function $q(t)$ is known (see Lemma 1.4 in [11]):

(1) If $t<1$, then $q(t)=0$, and all the derivatives of $q(t)$ are bounded functions on $\mathbb{R}$.

(2) Set

$$
\lambda(s)=\int_{0}^{\infty} t^{s-1} q(t) d t \quad(\operatorname{Re}(s)<0) .
$$

Then the function $\lambda(s)$ is a holomorphic function in the domain $\{s \in \mathbb{C} \mid \operatorname{Re}(s)<0\}$ and for every pair of positive numbers $\left(\nu_{1}, \nu_{2}\right)$, there exists a constant $c>0$ such that $|\lambda(s)|>c \exp (-\sqrt{|\operatorname{Im}(s)|})$ on $-\nu_{1} \leq \operatorname{Re}(s) \leq-\nu_{2}$. 
Set

$$
K_{\epsilon}=\left\{x \in V_{\epsilon}|| x_{m+1}|>1,| Q(x) \mid=1\right\}
$$

and take a function $h_{\epsilon} \in C_{0}^{\infty}\left(K_{\epsilon}\right)$ such that $h_{\epsilon} \geq 0$ and

$$
\int_{K_{\epsilon}} h_{\epsilon}(x) \omega(x)=1
$$

with $d x=d Q \wedge \omega$. For a positive integer $L$, let

$$
f_{\epsilon, L}(x)= \begin{cases}\left|x_{m+1}\right|^{m / 2-c(\epsilon, k)}|Q(x)|^{-L} q(|Q(x)|) h_{\epsilon}\left(x /|Q(x)|^{1 / 2}\right) & x \in V_{\epsilon}, \\ 0 & x \notin V_{\epsilon} .\end{cases}
$$

The function $f_{\epsilon, L}$ belongs to $C^{\infty}\left(V_{\mathbb{R}}\right)$. Then, by Lemma 4.5 in [14], Theorem 2.1 and the Poisson summation formula hold for $f_{\epsilon, L}$ for $L$ sufficiently large. Hence the following identity, which is derived from Propositions 3.5 and 3.6, still remains true for $f_{\epsilon, L}$ for $L$ sufficiently large.

$$
\begin{aligned}
\Lambda_{\tilde{D}}\left(s ; k, L_{\epsilon}\right)+\frac{a_{\epsilon}(k ; 0)}{s}+\frac{b_{\epsilon}(k ; 0)}{c(\epsilon, k)+1-s} \\
=\frac{\tilde{D}^{s / 2} \Gamma(s) B(s)}{(2 \pi)^{s} \Phi_{\epsilon}\left(f_{\epsilon, L} ; c(\epsilon, k)-\frac{m}{2}, s-c(\epsilon, k)-1\right)} \\
\quad-\frac{1}{s}\left(\frac{\tilde{D}^{s / 2} \Gamma(s) \zeta\left(c(\epsilon, k)-\frac{m}{2}+1\right) \Phi_{\epsilon}^{*}\left(\hat{f}_{\epsilon, L} ; c(\epsilon, k)-\frac{m}{2}, 0\right)}{(2 \pi)^{s} \Phi_{\epsilon}\left(f_{\epsilon, L} ; c(\epsilon, k)-\frac{m}{2}, s-c(\epsilon, k)-1\right)}-a_{\epsilon}(k ; 0)\right) \\
\quad-\frac{1}{c(\epsilon, k)+1-s} \\
\quad \times\left(\frac{\tilde{D}^{s / 2} \Gamma(s) \zeta\left(c(\epsilon, k)-\frac{m}{2}+1\right) \Phi_{\epsilon}\left(f_{\epsilon, L} ; c(\epsilon, k)-\frac{m}{2}, 0\right)}{(2 \pi)^{s} \Phi_{\epsilon}\left(f_{\epsilon, L} ; c(\epsilon, k)-\frac{m}{2}, s-c(\epsilon, k)-1\right)}-b_{\epsilon}(k ; 0)\right),
\end{aligned}
$$

where

$$
\begin{aligned}
B(s)=Z_{+} & \left(f_{\epsilon, L}, \phi_{1} ; c(\epsilon, k)+\frac{m}{2}, s-c(\epsilon, k)\right) \\
& +Z_{+}^{*}\left(\hat{f}_{\epsilon, L}, \hat{\phi}_{1} ; c(\epsilon, k)+\frac{m}{2}, 1-s\right) .
\end{aligned}
$$

For $\nu>0$ the above function is bounded on any vertical strip with $|\operatorname{Im}(s)|<$ $\nu$, since the left hand side of the above function is holomorphic. Therefore it is sufficient to prove that the above function is bounded on any vertical strip with $|\operatorname{Im}(s)|>\nu$. Then $Z_{+}\left(f_{\epsilon, L}, \phi_{1} ; c(\epsilon, k)+m / 2, s-c(\epsilon, k)\right)+$ 
$Z_{+}^{*}\left(\hat{f}_{\epsilon, L}, \hat{\phi}_{1} ; c(\epsilon, k)+m / 2,1-s\right)$ are bounded on any vertical strip and from Stirling's estimate, we have

$$
\Gamma(s)=O\left(|\operatorname{Im}(s)|^{\operatorname{Re}(s)-1 / 2} e^{-\pi|\operatorname{Im}(s)| / 2}\right)
$$

on any vertical strip. Therefore we need an estimate of $\Phi_{\epsilon}$ from below. By the definition of $f_{\epsilon, L}$, we obtain

$$
\begin{aligned}
\Phi_{\epsilon}\left(f_{\epsilon, L} ; c(\epsilon, k)-\frac{m}{2}, s-1\right) & =\int_{V_{\epsilon}}\left|x_{m+1}\right|^{c(\epsilon, k)-m / 2}|Q(x)|^{s-1} f_{\epsilon, L}(x) d x \\
& =\int_{0}^{\infty} t^{s-L-1} q(t)\left(\int_{K_{\epsilon, t}} h_{\epsilon}\left(x / t^{1 / 2}\right) \omega(x)\right) d t \\
& =\int_{0}^{\infty} t^{s-L-m / 2-1} q(t) \int_{K_{\epsilon}} h_{\epsilon}(x) \omega(x) d t \\
& =\int_{0}^{\infty} t^{s-L-m / 2-1} q(t) d t=\lambda\left(s-L-\frac{m}{2}\right),
\end{aligned}
$$

where

$$
K_{\epsilon, t}=\left\{x \in V_{\epsilon}|| x_{m+1}\left|>t^{1 / 2},\right| Q(x) \mid=t\right\} .
$$

Hence we obtain $\Phi_{\epsilon}\left(f_{\epsilon, L} ; c(\epsilon, k)-m / 2, s-1\right)>C e^{-\sqrt{|\operatorname{Im}(s)|}}(\operatorname{Im}(s) \rightarrow \pm \infty)$ and

$$
\begin{aligned}
& \Lambda_{\tilde{D}}\left(s ; k, L_{\epsilon}\right)+\frac{a_{\epsilon}(k ; 0)}{s}+\frac{b_{\epsilon}(k ; 0)}{c(\epsilon, k)+1-s} \\
& \quad=O\left(|\operatorname{Im}(s)|^{\operatorname{Re}(s)-1 / 2} e^{-\pi|\operatorname{Im}(s)| / 2+\sqrt{|\operatorname{Im}(s)|}}\right) .
\end{aligned}
$$

In particular, the function on the left hand side is bounded on any vertical strip. Our assertion for $\Lambda_{\tilde{D} r^{2}}\left(s ; k, L_{\epsilon}, \psi\right)$ can be proved in the same way as in the case of $\Lambda_{\tilde{D}}\left(s ; k, L_{\epsilon}\right)$. Therefore the functions $\Lambda_{\tilde{D}}\left(s ; k, L_{\epsilon}\right)$ and $\Lambda_{\tilde{D} r^{2}}\left(s ; k, L_{\epsilon}, \psi\right)$ satisfy all the conditions in the converse theorem.

Remark 3. Theorems 4.1 and 4.5 include several earlier results as special cases.

(1) In the case $m=1$ and $A=(1)$, the zeta functions were studied in [11] and the corresponding modular forms are the Cohen Eisenstein series.

In the case $m=2,(p, q)=(0,2)$ and

$$
A=\left(\begin{array}{cc}
-1 & 0 \\
0 & d_{\mathbf{K}} / 4
\end{array}\right) \quad \text { or } \quad A=\left(\begin{array}{cc}
-1 & -1 / 2 \\
-1 / 2 & \left(d_{\mathbf{K}}-1\right) / 4
\end{array}\right)
$$


according as $4 \mid d_{\mathbf{K}}$ or $4 \nmid d_{\mathbf{K}}$, where $d_{\mathbf{K}}$ is the discriminant of imaginary quadratic field $\mathbf{K}$. Theorems 4.1 and 4.5 have been obtained in [14].

(2) In the case $m=2,(p, q)=(1,1)$ and

$$
A=\left(\begin{array}{cc}
0 & 1 / 2 \\
1 / 2 & 0
\end{array}\right)
$$

then $-\operatorname{det} 2 A=1$ and the theorems give classical examples. In fact, our zeta function coincides with $\zeta(w-1) \zeta(s) \zeta(s+w-1) \zeta(w)^{-1}$ up to a constant and the corresponding modular forms is the holomorphic Eisenstein series.

(3) The functional equation of Theorem 4.1 was obtained in [5] under the assumption that $A$ be positive definite. Moreover he posed a conjecture that the zeta functions are related to modular forms. Theorem 4.5 gives an affirmative answer to this conjecture.

\section{REFERENCES}

[1] H. Cohen, Sums involving the values at negative integers of L-functions of quadratic characters, Math. Ann., 217 (1975), 271-285.

[2] R. Hörmander, An introduction to complex analysis in several variables, NorthHolland, 1973.

[3] T. Miyake, Modular forms, Springer, 1989.

[4] M. Muro, A note on the holonomic system of invariant hyperfunctions on a certain prehomogeneous vector space, Algebraic Analysis, vol. 2 (M. Kashiwara and T. Kawai, eds.), Academic Press (1988), pp. 493-503.

[5] M. Peter, Dirichlet series in two variables, J. reine angew. Math., 522 (2000), 27-50.

[6] F. Sato, Zeta functions in several variables associated with prehomogeneous vector spaces I: functional equations, Tohoku Math. J., 34 (1982), 437-483.

[7] F. Sato, Zeta functions in several variables associated with prehomogeneous vector spaces II: a convergence criterion, Tohoku Math. J., 35 (1983), 77-99.

[8] F. Sato, On functional equations of zeta distributions, Adv. Studies in pure Math., 15 (1989), 465-508.

[9] F. Sato, L-functions of prehomogeneous vector spaces, preprint (2003).

[10] G. Shimura, On modular forms of half integral weight, Ann. of Math., 97 (1973), 440-481.

[11] T. Shintani, On Dirichlet series whose coefficients are class-numbers of integral binary cubic forms, J. Math. Soc. Japan, 24 (1972), 132-188.

[12] T. Shintani, On zeta-functions associated with the vector space of quadratic forms, J. Fac. Sci. Univ. Tokyo, Sect. IA 22 (1975), 25-65.

[13] H. M. Stark, L-functions and character sums for quadratic forms (I), Acta Arithmetica, XIV (1968), 35-50. 
[14] T. Ueno, Elliptic modular forms arising from zeta functions in two-variable attached to the space of binary hermitian forms, J. Number Theory, 86 (2001), 302-329.

[15] A. Weil, Über die Bestimmung Dirichletscher Reihen durch Funktionalgleichungen, Math. Ann., 168 (1967), 149-156.

Department of Mathematics

Rikkyo University

Nishi-Ikebukuro

Tokyo, 171-8501

Japan

ueno@rkmath.rikkyo.ac.jp 